\title{
An unequal redundancy level-based mechanism for reliable data collection in wireless sensor networks
}

\author{
Qi Zhang and Anfeng Liu*
}

\begin{abstract}
Most applications require high reliability with low delay in wireless sensor networks (WSNs). Unfortunately, it is a challenge issue to achieve reliable communication because of the lossy nature of wireless channels. In this paper, a novel unequal redundancy level (URL) data collection scheme is proposed to achieve high reliability for wireless sensor networks while long lifetime can be still guaranteed. Aiming at the imbalance of energy consumption in WSNs, a URL data collection scheme adopts network coding-based mechanism with high redundancy level in non-hotspot areas while adopting low redundancy level in hotspot areas. The theoretical analysis and experimental results show that, comparing with equal redundancy level (ERL) approach, the URL approach improves the lifetime by $15-50 \%$ under the same reliability, and it can also improve the reliability by $14.1-28.3 \%$ under the same lifetime. Comparing with an SW-ARQ approach, the URL approach improves the lifetime by 7-30\% under the same reliability, and it can also improve the reliability by $8-23 \%$ under the same lifetime.
\end{abstract}

Keywords: Wireless sensor networks, Network coding, Delay, Reliability, Lifetime

\section{Introduction}

Wireless sensor networks (WSNs) are emerging as a promising platform that enables a wide range of applications in both military and civilian domains such as battlefield surveillance, medical monitoring, biological detection, etc. [1-7]. Due to their inherent characteristics of resourceconstraint, for data collection, energy efficiency is another vital issue since replacing or recharging the batteries of sensor nodes is extremely difficult [1-4]. In addition, some sensing applications require that each data packet is successfully delivered to sink with statistical probability such as $70-95 \%[1,8-11]$, which is sufficient for these applications such as environment (temperature, humidity) and agriculture (water tank, irrigation) $[1,10]$. Some reports reveal that the packet error rate of wireless links may be as high as $30 \%$, which is far from being satisfactory in practical wireless scenarios [11]. Furthermore, the specific incidents sensed by sensor nodes may exert "alarm" packets, which should be routed to sink by a certain action $[7,8]$. There might be such a case, for example, human intrusion

* Correspondence: afengliu@mail.csu.edu.cn

School of Information Science and Engineering, Central South University, Changsha 410083, China

\section{Springer Open}

(c) The Author(s). 2016 Open Access This article is distributed under the terms of the Creative Commons Attribution 4.0 International License (http://creativecommons.org/licenses/by/4.0/), which permits unrestricted use, distribution, and reproduction in any medium, provided you give appropriate credit to the original author(s) and the source, provide a link to the Creative Commons license, and indicate if changes were made. detection or fire detection in a large scale in WSNs [8]. In these situations, it is crucial to transfer the alert message to sink rapidly so that corresponding measures could be taken to respond to emergencies, for the tardy information can result in severe consequences like casualties or property loss $[8,9]$. Summarily, energy efficiency, the delay, and data reliability are regarded as the major concerns in the design of WSNs. There is a great deal of research that shows that the delay, energy efficiency, and data reliability have a trade-off $[1,7,8,10]$.

In WSNs, a data packet can be delivered from a sensor node to the sink node by means of multi-hop transmission [8]. Due to the lossy nature of wireless channels, a packet could be lost. In order to ensure high reliability, researchers have put forward a considerable mechanism, which can be divided into three categories:

(1)Data retransmit mechanism [1, 8, 10]. A packet can be retransmitted when the sender node cannot detect the reception of the receiver node within a predefined time. However, the sender 
may retransmit a packet repeatedly until it receives the acknowledgement message from the receiver or the number of retransmissions over the upper limit. This mechanism has the advantage of longer network lifetime and less energy consumed by data transmission. But the disadvantage of this mechanism is that the delay, especially in the multi-hop sensor networks, no matter using end-to-end acknowledgment or hop-by-hop acknowledgment, is very long. So this mechanism is limited in some delay-sensitive applications [1, 8, 10, 12-15].

(2) Redundancy-based retransmission mechanism. According to the reliability theory, the number of transmissions for the sender transmitting a data packet can be calculated. It does not need to receive the acknowledgement message from the receiver any more. The advantage of this mechanism is that the delay is short, but the energy consumption is large and this affects the application of this mechanism in real life [1, 10, 12-14].

(3) Network coding-based redundant protocol. It employs a network coding-based approach to improve the packet redundancy level when a link is unreliable. This method can be able to ensure the reliability of network for paying some extra energy consumption. It does not need to retransmit the data packet, so it can achieve a compromise optimization between the delay and energy consumption. So it is a better method [8].

To the best of our knowledge, although the above methods can make a good effect on improving network performance, they still could not achieve a tradeoff optimization among the network lifetime, data reliability and the delay. In this paper, based on the network coding-based redundant protocol [8], we proposed an unequal redundancy level (URL) data collection scheme, which can achieve the simultaneous optimization on the performance (network lifetime, data collection, the delay). And the main contributions of this paper are as follows:

1. The URL data collection scheme employs an adaptive redundancy-based approach to provide higher reliability and longer lifetime according to the application requirements. In URL scheme, a high redundancy level network coding-based approach is used in non-hotspot areas to reduce delay as well as enhancing the reliability at cost of large extra energy consumption. In WSNs, the areas near to sink taking more data forwarding, it costs large energy for the nodes in these areas and forms the so-called energy hole, which leads to the network death ahead of schedule. Studies have pointed out that when a WSN die, there is also rest as much as $90 \%$ of energy $[3,9]$. So, in the URL scheme, we take full advantage of rest energy in non-hotspot areas, improving the nodes' redundancy level in these areas and the network reliability will be enhanced as well; in the meantime, we decrease the nodes' redundancy level in hotspot areas to reduce their energy consumption and improve the network lifetime. In this paper, the node's redundancy level means the number of redundant packets that the node carried in order to improve the reliability. On the whole, the scheme we proposed can improve both the reliability and the lifetime. To the best of our knowledge, URL scheme is the first data collection strategy that can optimize multiple performances at the same time. This is also an important innovation of URL scheme which we proposed in this paper.

2. Through our extensive theoretical analysis and simulation experiment study, we demonstrate that for URL scheme, the high reliability, low delay and high lifetime can be achieved simultaneously. Comparing with the prior research, URL scheme has two advantages. (a) It takes full advantage of the residual energy and its energy utilization can achieve more than $85 \%$, which is several times to the original strategy. (b) It can also improve the quality of the reliability and lifetime significantly, which is difficult to be achieved in the past research. Compared to ERL scheme, under the same reliability, URL approach can improve the lifetime by $20-44.4 \%$, and under the same lifetime, the reliability can be improved by 14.1-28.3 \%. And under these two conditions, the delays are all increased by about $5 \%$. Compared to SW-ARQ approach, it improves the lifetime by $7-$ $30 \%$ under the same reliability, and it can also improve the reliability by $8-23 \%$ under the same lifetime. In the meantime, the delay of URL is greatly reduced. The URL data collection scheme has extensive applicability. It owns all the advantages that the network coding-based mechanisms have. It can be applied to varieties of network coding-based approach and can improve the performance of these methods significantly. So the proposed scheme has a very good meaning.

The rest of this paper is organized as follows: In section 2, the related works are reviewed. The system model and problem statement are described in section 3. In section 4, URL data collection scheme is presented with reliable guarantee and low delay for WSNs. The performance analysis of URL scheme is provided in section 5 . The analysis and comparison of the experiment 
result are in section 6 , and we present our conclusions in Section 7.

\section{Related work}

There are a lot of studies to guarantee the reliability in WSNs. We can divide them into the following three categories:

(a) Retransmission-based mechanism: The scenario is different in WSNs where packets are forwarded via multiple wireless hops. On each wireless link, the packet error rates (PER) are commonly 10-30 \% $[1,10]$, which significantly decrease the end-to-end reliability (the probability that a packet transmits successfully from the sender to the base station). Automatic Repeat-Request (ARQ) is an important mechanism to protect the reliability of data transmission [1, 10, 12-14]. There are three kinds of basic schemes: (1) send-and-wait (SW) protocol, (2) go-back-N (GBN) protocol, and (3) selective repeat (SR) [10, 12-14]. All the three ARQ protocols can operate in hop-by-hop $(\mathrm{HBH})$ or end-to-end (E2E) mode [10, 14]. With SW, the transmitter waits for an ACK or a timeout before its next transmission [10,14]. With GBN and SR, the transmitter sends packets continuously. However, to prevent buffer overflow at the receiving node, the number of unacknowledged transmissions is kept below a preset size of $\mathrm{N}$ packets [10]. GBN and SR are implemented by a sliding window of size $\mathrm{W}$, where $\mathrm{W}$ is determined by $\mathrm{N}$ and the round trip time (RTT) estimator [10]. The objective is to keep a continuous stream of transmissions, hence utilizing channel capacity while maintaining lower packet delay. The advantage of retransmission-based mechanism is the packet error rates get lower. As long as a packet is received, the sender can receive the ACK with a high probability. So the retransmissionbased mechanism can ensure a high reliability for receiver to receive data by retransmission more than once and the energy consumption of it is little. The protocol is easy to implement and suitable for various kind of routing protocols [10, 12-14]. Under this protocol, a sensor node end to send a packet when it has received an ACK from the receiver or the retransmission times exceed the threshold. So the disadvantage of this protocol is the network latency is very big, especially in a link of high packet error rates [10].

(b)Network coding techniques. Network coding techniques are reliability mechanism, which are based on the redundant coding. In the network coding techniques, the source node encodes the packets at the application layer. The destination node decodes the packets to retrieve the original packets. The re-ending/decoding operation at intermediate nodes is optional. Because of the redundant packets that the source node had, so it would not affect the destination, nodes retrieve the right data packets even if there are some data lost through the lossy nature wireless channels. The advantage of the network coding techniques is that it can one-time send all the data packets out and the destination node can receive the data packets by a higher reliability. So, relative to the retransmission-based mechanism, the delay will be greatly decreased. But in this approach, the nodes need to encode and decode the data packets, and store more redundant data, so it has a higher requirement for computing and storage capacity. A data collection protocol has proposed by Keller et al. [16], which combining the redundancy and network coding to achieve higher reliability under the highly dynamic network conditions. For this protocol, each node has to store the packets that it overhears, and this is a challenge for an energy-limited node. Because it requires coding at the intermediate nodes, so the overhead of delay and computational are all not solved. As a result, in terms of the end-to-end delay, the coding overhead has become a bottleneck problem. Through separating the coding and radio into two concurrent threads, the end-to-end delay is shortened, this is the protocol which is proposed by Gao et al. [17]. Pang et al. [18] have proposed a distributed network coding (DNC) approach, which the relay nodes send data to the destination node by performing network coding. Xu et al. [19] have proposed a collaborative data collection scheme, which employing opportunistic in-network recoding in a disruptive network environment [20].

(c) Forward error correction (FEC). It has been used to improve the network reliability in a lot of studies [21]. FEC can guarantee the delivery reliability by the redundancy of the small encoded block, and it improves the reliability by encoding the packets together. Thus, the network coding approach is better especially when the link loss rate is large.

And there are also several protocols which proposed to reduce the end-to-end delay in WSNs [22-25]. A crosslayer protocol proposed by Wang et al. [26] providing Qos support for the latency-sensitive traffic flows. It proposed a higher priority channel access and used a short frame structure to reduce the end-to-end delay. Shanti and 
Sahoo [27] have proposed delay guaranteed routing and MAC (DGRAM) for delay-sensitive applications in WSNs. DGRAM can provide deterministic delay guarantee and it uses slot reuse to reduce the latency. But it is vital to calculate the transmission and reception slots of nodes accurately. Because of basing on the cross layer, the above protocols [26, 27] are all difficult to implement.

The previous work has made a great foundation for this article's research. In the early studies, they are all do not optimize the performance from the perspective of the whole network, so when we want to optimize one performance, it also at the cost of reduction the other performances. For example: retransmission-based mechanism has good energy efficiency, but the delay is very long; the delay of the network coding techniques and forward error correction is short, but the energy efficiency is not high. So, there is a tradeoff optimization relation between several network performance indexes, such as: data transmission reliability, energy efficiency, and network latency, and it is difficult to optimize all the performance at the same time. Therefore, the URL scheme which is proposed in this paper can optimize the performance from the whole network, and it can optimize the network performance simultaneously in premise of ensuring the network reliability meets the application requirements.

\section{The system model and problem statement}

\subsection{The network model}

The network model used in this paper can also be seen in ref. [8] and ref. [9], that is, (1) $\lambda$ homogenous sensors are deployed in a circular region while the sink (base station) situated at the center. The node distribution follows a homogenous Poisson point process with a density of $\rho$ sensors per unit area; (2) the transmitting radius of sensor node is denoted by $r$. In the paper, we consider the probability of generation one date packages as $\lambda$ in a sample cycle [9], and this is refereed to one round. The sensory data is sent to the base station adopting greedy geographic routing [18]. (3) For each node $i$, the probabilities of successful transmission between node $i$ to node $i+1$ are denoted by $p_{i}$. Following ref. [10], we assume that reception failures are spatial dependent but time independent $[1,10,22-24]$.

\subsection{Energy consumption model and related definitions}

We adapt the typical energy consumption model $[9,22$, 28-30], the energy consumption of data transmission is shown in Eq. (1) and the energy consumption for data receiving is shown in Eq. (2).

$$
\begin{aligned}
& \left\{\begin{array}{l}
E_{\text {member }}=l E_{\text {elec }}+l \varepsilon_{f f} d^{2} \quad\left(d<d_{0}\right) \\
E_{\text {member }}=l E_{\text {elec }}+l \varepsilon_{\text {amp }} d^{4} \quad\left(d>d_{0}\right)
\end{array}\right. \\
& E_{R}(l)=l E_{\text {elec }}
\end{aligned}
$$

$E_{\text {elec }}$ represents the energy consumption in the transmission circuit. $d$ represents the transmission distance. If the transmission distance $d$ is less than the threshold $d_{0}$, the energy consumption caused by power amplification adopts the free space model; if the transmission distance is greater than or equal to the threshold, use the multipath fading model. $\varepsilon_{f s}$ and $\varepsilon_{a m p}$ are the energy required to amplify in the models, respectively. I represents the number of data bits. The specific set of the above parameters are taken from literature [1, 3, 9, 31-33], as shown in Table 1.

\subsection{Problem statement}

We consider multi-hop transmissions from a sensor node to the base station node (Sink). The problem that this paper studies can be described as: according to the application reliable requirement when the packets getting through each hop, how can each node select network coding redundancy level to minimize the delay, maximize the network lifetime and energy efficiency. The data collection routing of WSN can be characterized by several performance indicators as explained below:

(1)End to end delay for data collection routing (denoted as $D_{e 2 e}$ ). $D_{e 2 e}$ represents the time that from a packet originates to it transmit to sink. Let $d_{i}$ stands for the delay of the packets arrival at the $i^{\text {th }}$ hop of multi-hop routing to sink, then the end-toend delay minimization can be expressed as:

$$
\min \left(D_{e 2 e}\right)=\min \left(\sum_{i \text { croute }} d_{i}\right)
$$

(2)Effective energy utilization (denoted as $\zeta$ ). $\zeta$ refers to the ratio of energy efficiently utilized and the total energy in the network which can be expressed as a

Table 1 Parameters in the network

\begin{tabular}{ll}
\hline Parameter & Value \\
\hline Threshold distance $\left(d_{0}\right)(\mathrm{m})$ & 87 \\
$E_{\text {elec }}(\mathrm{nJ} / \mathrm{bit})$ & 50 \\
$\varepsilon_{f s}\left(\mathrm{pJ} / \mathrm{bit} / \mathrm{m}^{2}\right)$ & 10 \\
$\varepsilon_{\text {amp }}\left(\mathrm{pJ} / \mathrm{bit} / \mathrm{m}^{4}\right)$ & 0.0013 \\
Initial energy $\left(E_{\text {ini }}\right)(J)$ & 0.5 \\
\hline
\end{tabular}


formula below. In the formula, $e_{i}$ stands for the energy consumption of node $i, E_{i n i}$ is the initial energy of node $i$ :

$$
\max (\zeta)=\max \left(\left(\sum_{0 \leq i \leq n} e_{i}\right) /\left(\sum_{0 \leq i \leq n} E_{i n i}\right)\right)
$$

(3) Lifetime (denoted as $\ell$ ). Lifetime is defined as the death time of the first node in the network. So we should decrease the energy consumption of the biggest energy consumption node to maximum $\ell . e_{i}$ is the energy consumption of node $i, E_{\text {ini }}$ refers to the initial energy of each node. So we can describe the network lifetime as follows:

$\max (\ell)=\max \left(E_{\text {ini }} / e_{i}\right)$

(4) In this paper, we guarantee that the data collection reliability is higher than the reliability $\delta$, which is required by the application. $\delta_{e 2 e}^{j}$ refers to the reliability that a data packet send to sink from the node $j$. Let $\beta_{i}$ stand for the reliability for the packets at the $i^{t h}$ hop of multi-hop routing to sink, and then the end-to-end reliability can be expressed as:

$$
\underset{\substack{e 2 e \\ j \in\{1 . . n\}}}{\delta^{j}}=\prod_{i \in \text { path } j} \beta_{i} \geq \delta
$$

Obviously, the goal of URL scheme is that on the premise of ensuring the network reliability meet the application requirements, minimize the network latency and maximize the network lifetime and the energy efficiency, which can be stated as follows:

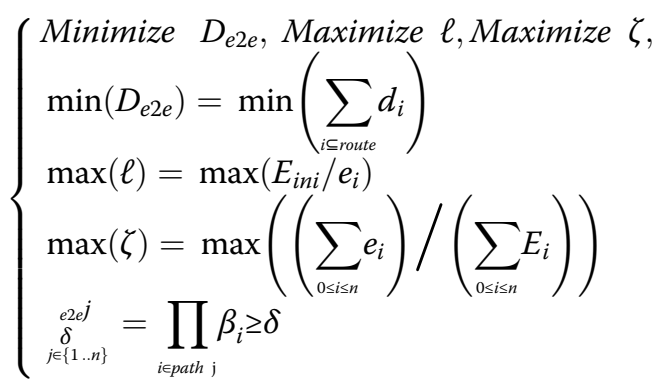

In order to make readers have a better understanding of this paper, the main notations introduced in this paper can be found in Table 2 .

\section{Unequal redundancy level (URL) approach design}

\subsection{Research motivation}

The research motivation of this paper mainly origins from the following two observations. The experiment parameters of Figs. 1, 2, 3, and 4 are presented in Table 3.

\subsubsection{Observation 1}

With high network coding based redundancy level, the reliability is higher, while the energy consumption is also increased $[8,10]$. Thus, there is a tradeoff between reliability and energy efficiency $[34,35]$. We confirm this situation by Figs. 1 and 2. Other things being the same, in Fig. 1, as the redundancy level increasing from 1 to 15 , the reliability also improves from 70 to $99.9 \%$. And in Fig. 2, when the node's redundancy level is growing, the data sizes and energy consumption are also increased. So we can find

\begin{tabular}{|c|c|}
\hline parameter & Meanings \\
\hline$p_{c}$ & Link loss rate \\
\hline$p_{e}$ & End-to-end reliability of data packets \\
\hline$m_{i}$ & $\begin{array}{l}\text { The redundancy level for nodes in the } i^{\text {th }} \text { loop under } \\
\text { URL scheme }\end{array}$ \\
\hline$m$ & $\begin{array}{l}\text { The nodes' redundancy level of each loop under ERL } \\
\text { scheme }\end{array}$ \\
\hline$p$ & The reliability to next hop under ERL scheme \\
\hline$p_{i}$ & $\begin{array}{l}\text { The reliability of the } i^{\text {th }} \text { loop to next hop under URL } \\
\text { scheme }\end{array}$ \\
\hline$\hat{N}$ & The amount of data of each packet in linear network \\
\hline$\hat{N}_{m}$ & $\begin{array}{l}\text { The number of data transmission to next hop in linear } \\
\text { network }\end{array}$ \\
\hline$\zeta_{1}^{e, t}$ & $\begin{array}{l}\text { The transmit data sizes for the nodes that are / distance } \\
\text { to sink under ERL scheme }\end{array}$ \\
\hline$\zeta_{1}^{e, r}$ & $\begin{array}{l}\text { The received data sizes for the nodes that are / distance } \\
\text { to sink under ERL scheme }\end{array}$ \\
\hline$E_{1}^{e}$ & $\begin{array}{l}\text { The energy consumption of the nodes that are / distance } \\
\text { to sink under ERL scheme }\end{array}$ \\
\hline$\zeta_{1}^{u, t}$ & $\begin{array}{l}\text { The transmit data sizes for the nodes that are / distance } \\
\text { to sink under URL scheme }\end{array}$ \\
\hline$\zeta_{1}^{u, r}$ & $\begin{array}{l}\text { The received data sizes for the nodes that are / distance } \\
\text { to sink under URL scheme }\end{array}$ \\
\hline$E_{1}^{u}$ & $\begin{array}{l}\text { The energy consumption of the nodes that are / distance } \\
\text { to sink under URL scheme }\end{array}$ \\
\hline$\phi$ & The percentage of enhancing the reliability \\
\hline$\Omega$ & The percentage of prolonging the network lifetime \\
\hline$R_{D_{U R L}}$ & The network latency incurred by URL scheme \\
\hline
\end{tabular}
that if we want to improve the network reliability, we

Table 2 Notations 


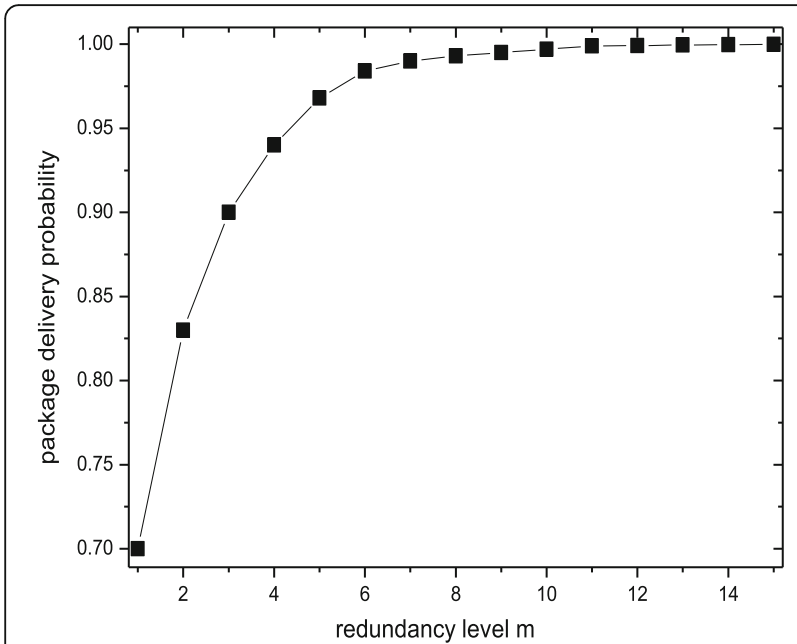

Fig. 1 Reliability improved by the redundancy level growing

should to improve the node's redundancy level, and it needs more energy consumption.

\subsubsection{Observation 2}

In the equal redundancy level (ERL) studies, there is a lot of residual energy in the areas far to sink. Thus, we are inspired to adopt high network coding based redundancy level in the areas where remained lots of energy. So we can greatly improve the data collection reliability and not reduce the network lifetime. If we decrease the redundancy level of the nodes in the regions near to sink, the network lifetime could also be improved.

Figure 3 presents the energy consumption under ERL scheme in the linear network. From experimental

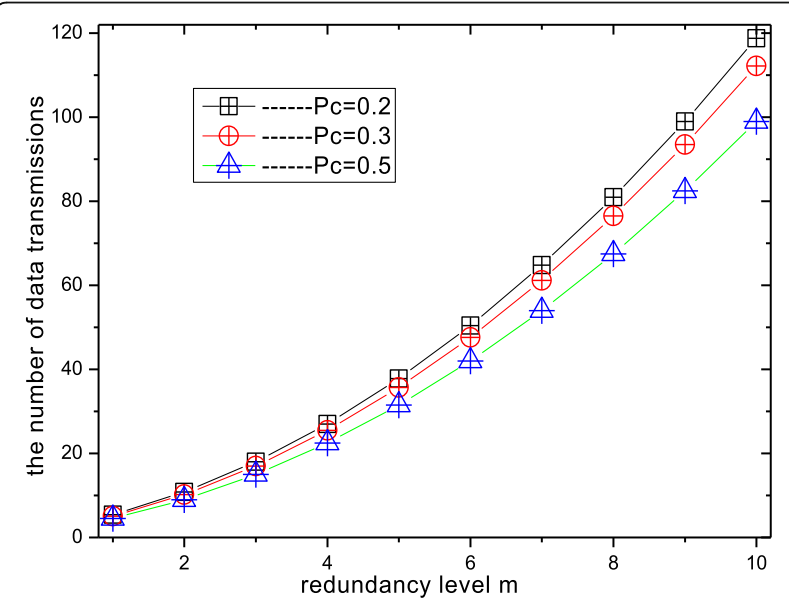

Fig. 2 Data sizes increased by the redundancy level growing

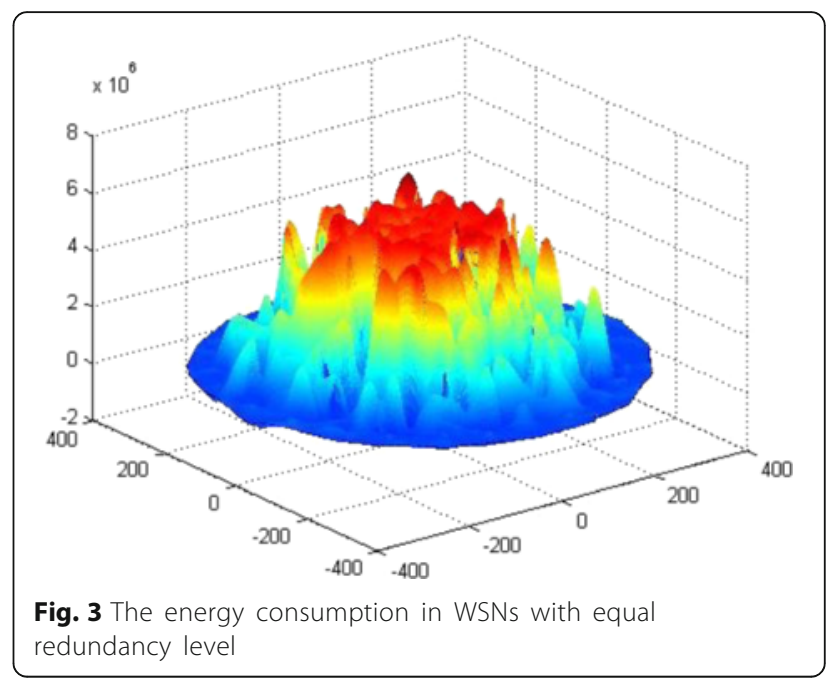

results, we can find that the nodes near to sink have a higher energy consumption, and the energy consumption is low for the nodes far to sink, the energy consumption of the entire network is imbalanced. Figure 4 shows the rest of energy in different areas in the network. From the graph, we can see that there are lots of rest energy in the areas far to sink, we call them as non-hotspot areas. And there are almost all the energy consumed in the areas near to sink, we call them hotspot areas. Studies have pointed that, when the network is dead, there is also over $90 \%$ of rest energy in the network $[2,6]$.

Based on above research, a new mechanism which is called URL scheme is proposed in this paper. The main idea of it is: we can get the end-to-end

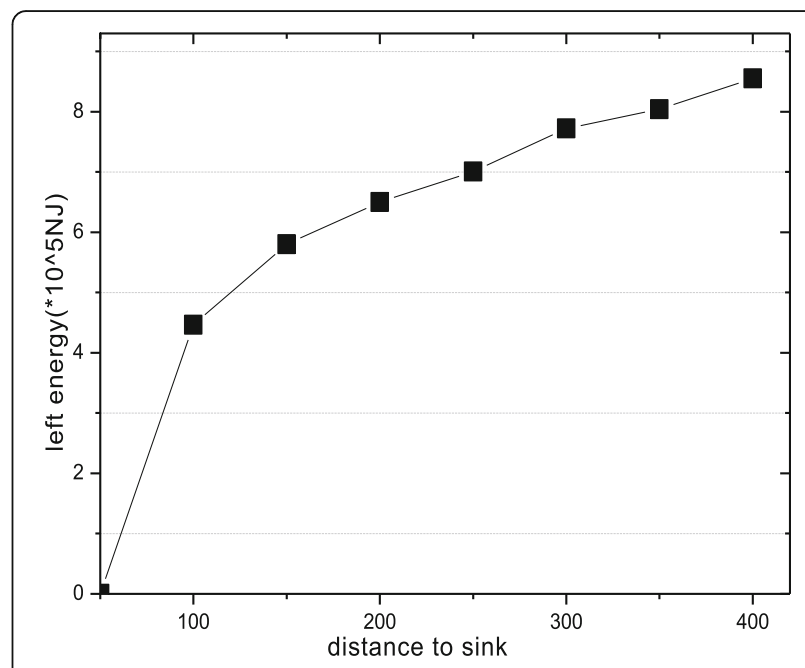

Fig. 4 The left energy in WSNs with the equal redundancy level 
Table 3 Experimental network scenarios

\begin{tabular}{ll}
\hline Sink node number & 1 \\
Sensor node number & 1000 \\
Network radius & $400 \mathrm{~m}$ \\
Nodal transmission radius & $50 \mathrm{~m}$ \\
$\begin{array}{l}\text { Initial energy for each node } \\
\text { The minimum end-to-end } \\
\text { reliability limit }\end{array}$ & $70 \%$ \\
$\begin{array}{l}\text { Dynamic routing } \\
\text { Others parameters }\end{array}$ & Shortest routing algorithm [22] \\
\hline
\end{tabular}

reliability by multiplying the hop-to-hop reliability. So, under a certain reliability $p_{e}$, we can increase the nodes' redundancy level in the areas far to sink and decrease the nodes' redundancy level in the areas near to sink; thus, the network performances are improved. Adopting URL scheme in the network, we can reduce the energy consumption in hotspot areas, prolonging the network lifetime. While the energy consumption is increased in non-hotspot areas, but the nodes in these areas have lots of rest energy, so the increase of the redundancy level could not influence the whole network lifetime.

First, we confirm the validity of the URL scheme. The experimental setup as follows: there are 10 nodes in a linear network and the end-to-end reliability that the applications required greater than or equal to $70 \%$. Figure 5 shows the redundancy level of each nodes under ERL scheme and URL scheme when satisfying the condition that the end-to-end reliability greater than or equal to $70 \%$. Figure 6 presents the reliability under ERL and URL scheme. The experimental results showing

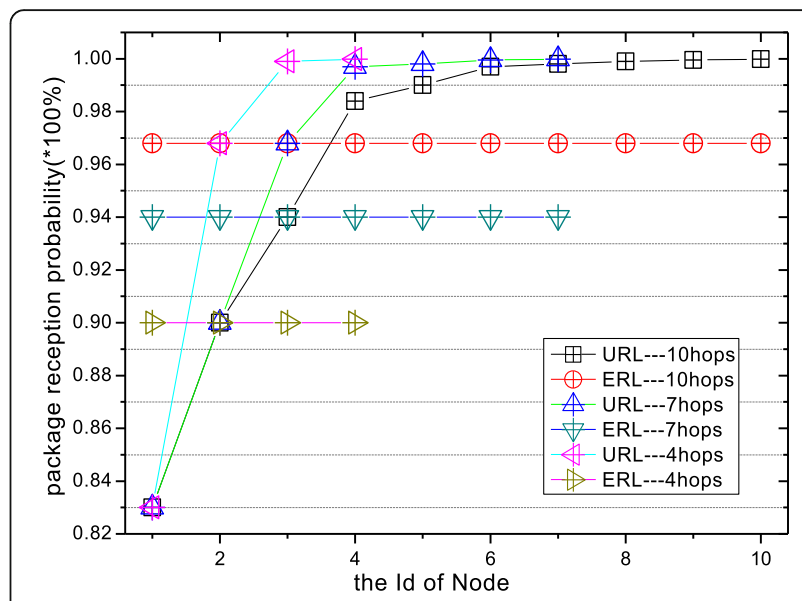

Fig. 6 The packet reception probability with different redundancy levels

that the URL scheme adopts low redundancy level near to sink while high redundancy level far to sink, thus the reliability to next hop of near to sink area is low and far to sink area is high, as Figs. 5 and 6 shows.

In Fig. 7, it presents the energy consumption of each node. The redundancy level of all the nodes is 5 in ERL scheme. But in the URL scheme, the redundancy level increased gradually by the position relative to the sink. Then, the nodes' redundancy level are, in order, 2, 3, 4, 6, 7, 8, 9, 10, 12, and 13. From the experimental results, we can find that the maximum energy consumption of URL scheme is less half than the energy consumption under ERL scheme, this can strongly suggest that the URL scheme could prolong the network lifetime substantially while ensuring the reliability.

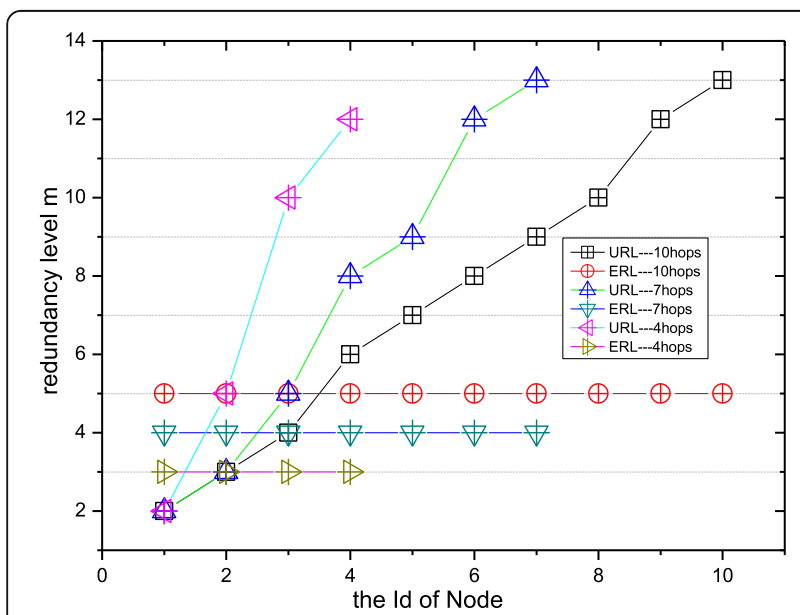

Fig. 5 The node with different redundancy levels

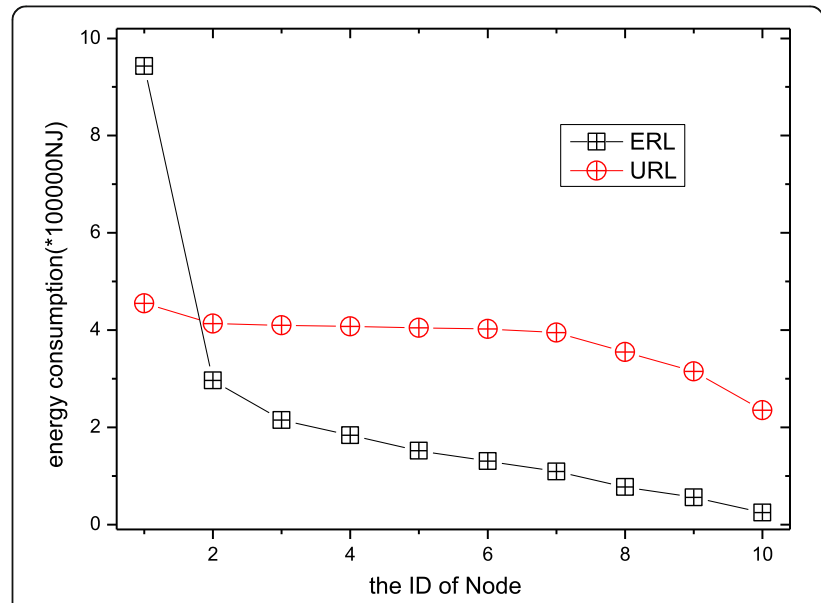

Fig. 7 Energy consume under different mechanisms (10 hops) 


\subsection{The URL scheme design}

This paper employs the same network coding techniques as ref. [8] to ensure the end-to-end network reliability greater than or equal to $p_{e}$. In network coding techniques, when the node's redundancy level is $m$ and the link loss rate is $p_{c}$, then the reliability $p$ for the node transmitting a data packet to next hop can denote as the following formula:

$$
p=\sqrt{1-p_{c}^{m+2}-(m+2) \cdot p_{c}^{m+1} \cdot\left(1-p_{c}\right)}
$$

In linear network, for each node that the redundancy level is $m$, the number of data transmissions generated by per packet is

$$
\hat{N}=\left(2-p_{c}\right) \times \frac{2+m}{2}
$$

Thus, if a node transmits the data packets to next hop with the reliability $p$, the formula for the amount of data transmissions is

$$
\hat{N}_{m}=\left(2-p_{c}\right) \times \frac{(m+1)(m+2)}{2}
$$

$m$ refers to the node's redundancy level, $p_{c}$ is the link loss rate.

Through the previous analysis, we can find that in the case of link loss rate is unchanged, the reliability and data transmissions are all have a positive correlation to the redundancy level. We also realize that the network lifetime lie on the nodes' lifetime which in hotspot areas. So in the URL scheme, we decrease the nodes' redundancy level in hotspot areas and the reliability to next hop in these areas is also decreased. In order to ensure the end-to-end reliability $p_{e}$, we increase the nodes' redundancy level in non-hotspot areas, and the reliability to next hop in these areas is increased. Thus, reducing the nodes' redundancy level in hotspot areas should meet the conditions that the nodes in non-hotspot areas have enough rest energy to improve the redundancy level to ensure the end-to-end reliability greater than or equal to $p_{e}$. We can determine the redundancy level in different areas by analyzing the relations between energy consumption and the reliability.
First, we analyze the amount of data transmissions in different areas and the reliability of data transmission when whole network use the same redundancy level. It has been proved in ref. [6] that, without considering the reliability, assume the probability of each node generating a data packet in a sample cycle is $\lambda$, the received data sizes for a node that is $l$ distance to sink can represent as the following formula:

$$
\begin{aligned}
\zeta_{l} & =\left(1+\frac{l+r}{l}+\frac{l+2 r}{l}+\cdots+\frac{l+z r}{l}\right) \lambda \mid l \\
& =h r+x, x+z r \leq R
\end{aligned}
$$

In ERL scheme, we assume the maximum hop is $\hbar$ and the application required minimum reliability is $\hat{p}$. Then, the reliability to next hop denote as $p_{o}$ should meet the following formula:

$$
\left(p_{o}\right)^{\hbar} \geq p_{e} \mid p_{o}=\sqrt{1-p_{c}^{m+2}-(m+2) \cdot p_{c}^{m+1} \cdot\left(1-p_{c}\right)}
$$

That is:

$$
\left(p_{o}\right)^{\hbar} \geq p_{e} \Rightarrow \sqrt{1-p_{c}^{m+2}-(m+2) \cdot p_{c}^{m+1} \cdot\left(1-p_{c}\right)} \geq \sqrt[n]{p_{e}}
$$

Thus, we can get the redundancy level $m$ under ERL scheme:

$$
1-p_{c}^{m+2}-(m+2) \cdot p_{c}^{m+1} \cdot\left(1-p_{c}\right) \geq \sqrt[h]{\left(p_{e}\right)^{2}}
$$

We take logarithm on the both sides in the above formula, and then we can get:

$$
m \log p_{c}+\log \left[p_{c}+(m+2)\left(1-p_{c}\right)\right]=\log \frac{\left(1-\sqrt[h]{\left(p_{e}\right)^{2}}\right)}{p_{c}}
$$

Because the value of $m$ is integer, so we can get an approximate value of $m$ according to formula (14) easily. And we can get the transmit data sizes for the nodes that are $l$ distance to sink is, 


$$
\begin{gathered}
\zeta_{l}^{e, t}=\left(\left(1+\frac{l+r}{l} p_{o}+\frac{l+2 r}{l}\left(p_{o}\right)^{2}+\cdots+\frac{l+z r}{l}\left(p_{o}\right)^{z-1}\right)\right. \\
(m+1)) \lambda \mid l=h r+x, x+z r \leq R
\end{gathered}
$$

The received data sizes for the nodes that are $l$ distance to sink is

$$
\begin{gathered}
\zeta_{l}^{e, r}=\left(\frac{l+r}{l} p_{o}+\frac{l+2 r}{l}\left(p_{o}\right)^{2}+\cdots+\frac{l+z r}{l}\left(p_{o}\right)^{z-1}\right) \\
(m+1) \lambda \mid l=h r+x, x+z r \leq R
\end{gathered}
$$

According to formulas (1) and (2), the energy consumption for the nodes that are $l$ distance to sink is

$$
E_{l}^{e}=E_{\text {member }}+E_{R}(l)=\zeta_{l}^{e, r} e_{u}^{r}+\zeta_{l}^{e, t} e_{u}^{t}
$$

We had calculated the energy consumption under ERL scheme. The initial state of URL scheme has the same parameters with ERL. On this basis, the main steps of URL scheme are as follows:

(1)In order to improve the reliability, first testing if we could improve the nodes' redundancy level in nonhotspot areas, that is, the nodes in the regions far to sink.

In this paper, if the nodes have the almost same distance to sink, we assume that the nodes have the almost same distance to sink are in the same loop. Because the energy consumption will increase if improving the nodes' redundancy level in the first loop or the second loop. So we should increase the nodes' redundancy level at least from the third loop. We improve the nodes' redundancy level in the $i^{\text {th }}$ loop, and now the redundancy level we can express in sequence as $m_{1}, m_{2}, \ldots m_{i}, \cdots m_{n}$. We can use formula (8) to calculate the nodal reliability $p_{1}, p_{2}, \ldots$ $p_{i}, \cdots, p_{n}$

Then, we calculate the energy consumption for the nodes that are $l$ distance to sink.

Let $\Gamma=\frac{l l}{r}$, then the redundancy level and the reliability for the nodes that are $l$ distance to sink can be expressed as $m_{\Gamma}$ and $p_{\Gamma}$.

At this moment, the transmit data sizes for the nodes that are $l$ distance to sink are

$$
\begin{aligned}
\zeta_{l}^{u, t}= & \left(\frac{l+r}{l} \cdot p_{\Gamma+1}+\frac{l+2 r}{l} \cdot p_{\Gamma+2} \cdot p_{\Gamma+1}+\cdots\right. \\
& \left.\quad+\frac{l+z r}{l} \cdot\left(p_{n} \cdots \cdot p_{\Gamma+1}\right)+1\right)\left(m_{\Gamma}+1\right) \lambda \mid l \\
= & h r+x, x+z r \leq R
\end{aligned}
$$

The received data sizes for the nodes that are $l$ distance to sink are:

$$
\begin{aligned}
\zeta_{l}^{u, r}= & \left(\frac{l+r}{l} \cdot p_{\Gamma+1}+\frac{l+2 r}{l} \cdot p_{\Gamma+2} \cdot p_{\Gamma+1}+\cdots\right. \\
& \left.\quad+\frac{l+z r}{l} \cdot p_{n} \cdots \cdot p_{\Gamma+1}\right)\left(m_{\Gamma+1}+1\right) \lambda \mid l \\
= & h r+x, x+z r \leq R
\end{aligned}
$$

According to formulas (1) and (2), the nodes' energy consumption under the URL scheme can be denoted as:

$E_{l}^{u}=E_{\text {member }}+E_{R}(l)=\zeta_{l}^{u, r} e_{u}^{r}+\zeta_{l}^{u, t} e_{u}^{t}$

If after improving the redundancy level for the nodes which in at least the third loop, the energy consumption in non-hotspot areas are all still less than the hotspot areas, then we can continue to improve the nodes' redundancy level in non-hotspot areas.

(2) Testing whether we can decrease the nodes' redundancy level in the first loop or the second loop.

After decreasing the nodes' redundancy level in hotspot areas, we should calculate the reliability in different areas again according to formula (8) and check the reliability at this time whether meeting the application required, that is whether meeting this type:

$p_{1} \times p_{2} \times \ldots p_{i} \times \cdots \times p_{n} \geq p_{e}$

If type (21) is founded, we can decrease the nodes' redundancy level in hotspot areas successfully, else we cannot decrease the redundancy level. Finally, return the first step testing again.

(3) The algorithm comes to the end when we cannot increase the nodes' redundancy level in the first step and decrease the nodes' redundancy level in the second step.

So the pseudo-code of URL scheme represents as algorithm 1. 
Algorithm1: obtain the redundancy level for each node which maximize the network lifetime and achieve high reliability;

Input: link loss rate $p_{c}$, package delivery probability $p_{e}$, network radius $R$, the node's initial energy $E_{\text {ini }}$ and relevant energy model parameters, the nodes' redundancy level $m$ when employing the ERL scheme, the nodal reliability $p$, nodal energy consumption $E_{i}^{\partial}$;

Output: nodes' redundancy level m;

1: Initialization: The nodal reliability : $p_{1}=p_{2}=p_{3}=\cdots=p_{n}=p$;

The nodes' redundancy level : $m_{1}=m_{2}=m_{3}=\ldots=m_{n}=m$;

$2: \mathrm{k}=0$;

3: $\operatorname{For}(0 \leq k \leq 2)$

4: $\quad k=k+1$;

5: $\quad$ While $\left(m_{k} \neq 0\right)$

6: $\quad m_{k}=m_{k}-1$;

7: $\quad$ According to formula (8), (20) calculate the nodal reliability $p_{k}$, nodes' energy consumption $E_{k}^{\partial}$ in the $k t h$ loop and nodes' energy consumption $E_{1}^{\partial}$ in the first loop;

8: $\quad$ while $\left(p_{1} p_{n} \ldots p_{n}<p_{e}\right)$

9: according to formula (20) we can find the minimum energy consumption node and set it as $i^{\text {th }}$ loop;

10: $m_{i}=m_{i}+1$

11: according to formula (8), (20) calculate the nodal reliability $p_{i}$ and the energy consumption $E_{i}^{\partial}$;

12: If $\left(E_{i}^{\partial}<E_{1}^{\partial} \& \& E_{i-1}^{\partial}<E_{1}^{\partial} \& \& m_{i}<50 \& \& p_{1} p_{2} \ldots p_{n}<p_{e}\right)$

13: $\quad$ goto 9 ;

14: $\quad$ Else

15: $\quad m_{i}=m_{i}-1$

16: End if;

17: End while;

18: Output: $m_{1}, m_{2}, m_{3}, \quad m_{n}$;

19: END 
The computational results are shown in Figs. 8, 9, 10, and 11. For ERL scheme, the nodes' redundancy level is equal. While in URL scheme, the nodes' redundancy level is high in the areas far to sink and low in the areas near to sink. Figure 8 shows the nodes' redundancy level under different schemes, and Fig. 9 presents the nodal reliability. We can see that the reliability for nodes in the areas near to sink is low and far to sink is high, so we can still guarantee the end-to-end reliability satisfy the applications required.

Figures 10 and 11 show the energy consumption and data sizes under these two different schemes. Because the URL scheme decreases the energy consumption in hotspot areas, so in premise of ensuring the end-toend reliability, the proposed scheme prolong the network lifetime. Although under URL scheme, the nodes' energy consumption is increased in nonhotspot areas, the energy consumption of these nodes is still less than the nodes' energy consumption in hotspot areas, so it has no influence on the whole network lifetime, it only make use of energy in the network more adequately.

\section{The performance analysis of the URL scheme 5.1 Reliability analysis}

Theorem 1 In the network that the radius is $R=\hbar \times r$, $m_{i}$ represents the nodes' redundancy level in the $i^{\text {th }}$ loop, $p_{c}$ represents the link loss rate, $p_{0}$ is the reliability to next hop under the ERL scheme, $\hbar$ refers to the network hops to sink. Under the circumstance that we guarantee the same lifetime for these two different mechanism, when we employing URL scheme, the percentage of the reliability performance we can improve at least

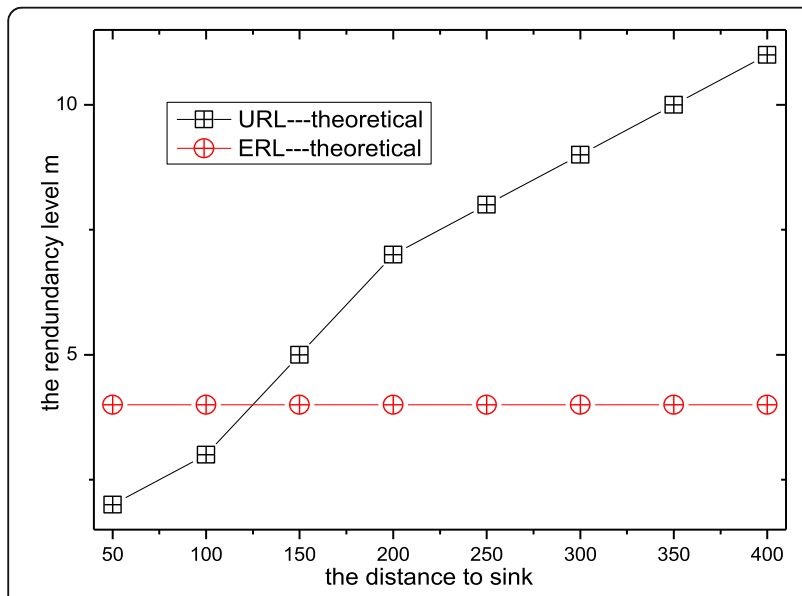

Fig. 8 Redundancy level for different mechanisms

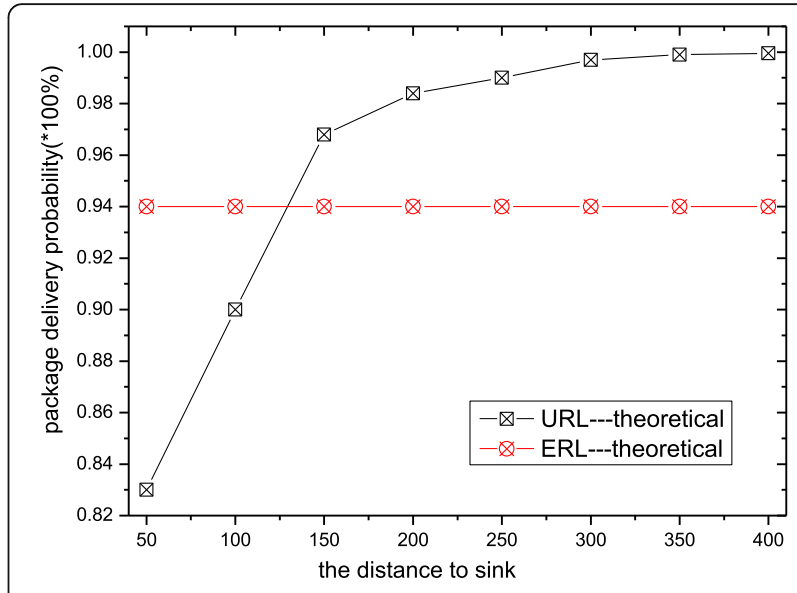

Fig. 9 Package delivery probability for different mechanisms

$$
\begin{aligned}
\phi & =\frac{p_{U R L}-p_{E R L}}{p_{E R L}} \\
& =\frac{p_{o}^{2} \prod_{i=3}^{\hbar} \sqrt{1-p_{c}^{m_{i}+2}-\left(m_{i}+2\right) \times p_{c}^{m_{i}+1} \times\left(1-p_{c}\right)}-p_{o}^{\hbar}}{p_{o}^{\hbar}} \times 100 \%
\end{aligned}
$$

Proof According to formula (8), we can calculate the reliability to next hop $\left(p_{o}\right)$ under ERL scheme. Because in ERL scheme, all the nodes have the same redundancy level, so they have the same reliability to next hop, and we have used $p_{o}$ to represent it. Then, the nodal reliability to sink can represent as:

$$
p_{E R L}=p_{o}^{\hbar}
$$

Adopting the URL scheme from formulas (18) and (19), we can find that it almost has no effect on the data sizes for the nodes in the first loop when we increasing the nodes' redundancy level in after the third loops. So

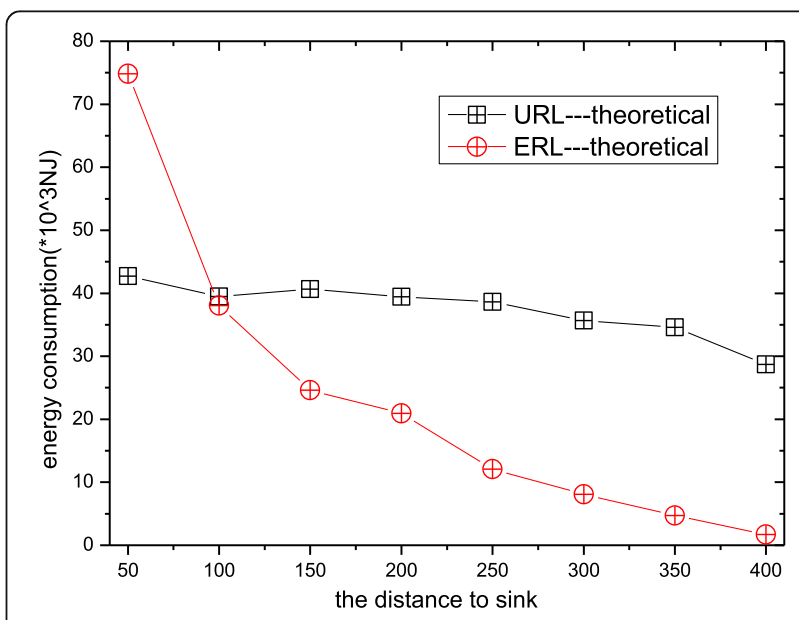

Fig. 10 Energy consumption under different mechanisms 


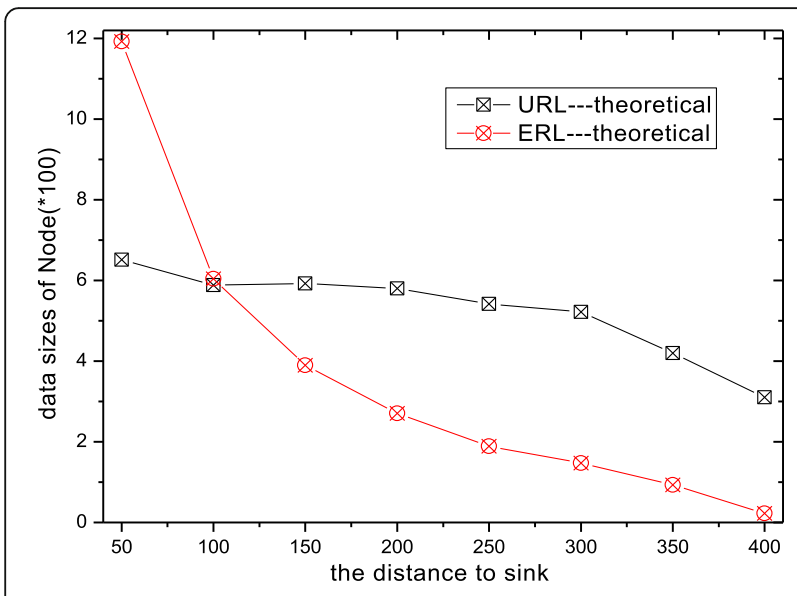

Fig. 11 Data sizes of node under different mechanisms

we can use all the rest energy to improve the nodes' redundancy level in after the third loops. In this way, we improve the end-to-end reliability in premise of ensuring the lifetime is unchanged. We can determine the increased of node's redundancy level by checking it's rest energy, and then calculate the percentage of the reliability has improved. According to the data sizes in formula (19), the received data sizes have a closely relation to the node's redundancy level of its next loop. So we should determine the nodes' redundancy level from the farthest area to sink. The energy consumption has a large gap between the outside loop and the inside loop, and by calculating the reliability, we can find that when the value of $m$ greater than 40 , the reliability to next hop almost equal to $100 \%$ under any link qualities. So for $m$ greater than 40 , there is no point in still increasing the value of it. In this paper, we assume that $m$ less than 50 satisfies the required precision of application.

Then after adjusting the nodes' redundancy level in the $(i+1)^{t h}$ loop, for the nodes in the $i^{t h}$ loop, the rest energy is

$$
E_{r, u}=E_{i n i}-\zeta_{i^{u, r}} e_{u}^{r}-\zeta_{i^{u, t}} e_{u}^{t}
$$

For the nodes in the $i^{\text {th }}$ loop, when we set $m_{i}=m_{i}+1$, according to formula (8), we can get a new reliability $p_{\hat{i}}$, the original reliability of these nodes is $p_{i}$, the increase of transmitting data sizes is

$$
\begin{array}{r}
\Delta_{i^{u, t}}=\left(\frac{l+r}{l} \cdot p_{i+1}+\frac{l+2 r}{l} \cdot p_{i+2} \cdot p_{i+1}+\cdots+\frac{l+z r}{l}\right. \\
\left.\cdot\left(p_{n} \cdots \cdot p_{i+1}\right)+1\right) \lambda \mid l=h r+x, x+z r \leq R
\end{array}
$$

Because if the nodes' redundancy level in the adjacent loops are unchanged, the received data sizes are almost unchanged. For the nodes in the $i^{\text {th }}$ loop, if the value of $m$ increase to $m+I$, then the increased energy consumption of these nodes is:

$$
E_{\Delta}^{i, u}=\Delta_{I}^{u, t} e_{u}^{t}
$$

So for the nodes in the $i^{\text {th }}$ loop, we can increase the number of redundant packets $\left(\eta_{i}\right)$ :

$$
\eta_{i}=\left\lceil\frac{E_{r}^{i, u}}{E_{\Delta}^{i, u}}\right\rceil
$$

By this time, the redundancy level for the nodes in the $i^{\text {th }}$ loop can represent as

$$
m_{i}=m_{i}+\eta_{i}
$$

The reliability to next hop for these nodes can be calculated by formula (8). Then, the network reliability is

$$
\begin{aligned}
p_{\text {URL }} & =p_{o}^{2} p_{3} p_{4} \cdots p_{i} p_{i+1} \cdots p_{n} \\
& =p_{o}^{2} \prod_{i=3}^{n} \sqrt{1-p_{c}^{m_{i}+2}-\left(m_{i}+2\right) \times p_{c}^{m_{i}+1} \times\left(1-p_{c}\right)}
\end{aligned}
$$

So, in a network where the radius is $\hbar_{r}$ the percentage of the reliability that URL scheme can improve is

$$
\begin{aligned}
\phi & =\frac{p_{U R L}-p_{E R L}}{p_{E R L}} \\
& =\frac{p_{o}^{2} \prod_{i=3}^{\hbar} \sqrt{1-p_{c}^{m_{i}+2}-\left(m_{i}+2\right) \times p_{c}^{m_{i}+1} \times\left(1-p_{c}\right)}-p_{o}^{\hbar}}{p_{o}^{\hbar}} \times 100 \%
\end{aligned}
$$

According to the theorem 1, we can see that the URL scheme improves the network reliability by increasing the nodes' energy consumption in non-hotspot areas, and it also guarantees the nodes' energy consumption in hotspot areas are unchanged. So the mechanism can improve the reliability of network data transmission and guarantee the network lifetime is unchanged. Figures 12 and 13 show in linear network that the reliability $p_{e}$ greater than or equal to $70 \%$, the same network lifetime is guaranteed, the energy consumption under different link loss rate $\left(p_{c}=0.3\right.$ and $\left.p_{c}=0.5\right)$. Table 4 presents the relevant parameters of SW-ARQ approach. It is observed that when employing URL scheme, the energy consumption in the regions far to sink is much larger than that under ERL and SW-ARQ approach, so the nodes' redundancy level is improved in the areas far to sink, the whole network reliability is also improved.

Figure 14 shows the reliability under three methods in the premise of ensuring the same lifetime. As can be seen from the calculation results, the reliability in nonhotspot areas under URL scheme is much higher than those under ERL and SW-ARQ approaches. This shows 


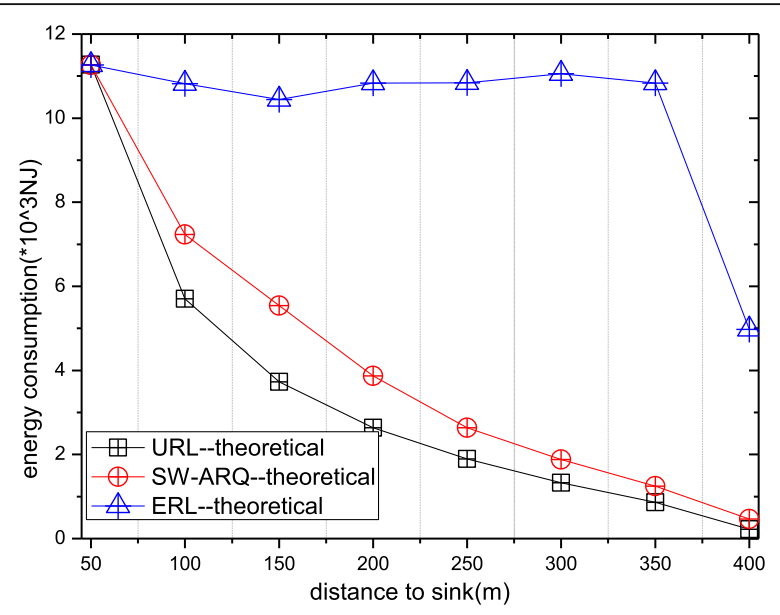

Fig. 12 Energy consumption for different mechanisms $\left(p_{e}=0.3\right.$, $\hat{p} \geq 70 \%)$

that the URL scheme can increase the reliability by a large margin in premise of ensuring the network lifetime is unchanged.

Figure 15 presents the enhance percentage of reliability for the whole network in different link qualities when employing URL scheme under the condition that guarantee the same lifetime and the end-to-end packet reception rate $p_{e} \geq 70 \%$. As a result, in premise of ensuring the network lifetime, compared with ERL scheme, the URL scheme enhances the end-to-end reliability by 12 to $31 \%$; compared with SW-ARQ approach, the URL scheme enhances the end-to-end reliability by 7 to $26 \%$. And for the proposed mechanism in this paper, the worse the link quality is, the higher the data transmission reliability is.

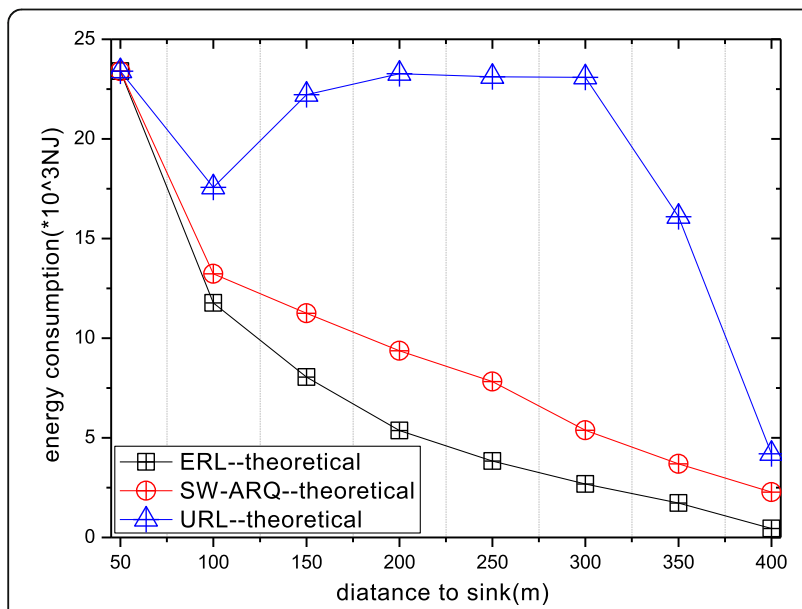

Fig. 13 Energy consumption for different mechanisms $\left(p_{e}=0.5\right.$, $\hat{p} \geq 70 \%)$
Table 4 Related parameters setting in SW-ARQ approach

\begin{tabular}{ll}
\hline Parameter & Value \\
\hline Length of ACK & 20 bits \\
Round-trip time $t_{r t t}$ & $200 \mathrm{~ms}$ \\
Retransmission time out $t_{\text {rto }}$ & $250 \mathrm{~ms}$ \\
Waiting time $\Delta t$ & $50 \mathrm{~ms}$ \\
Interval & $5 \mathrm{~ms}$ \\
\hline
\end{tabular}

\subsection{Lifetime analysis}

Theorem 2 In the URL scheme, under the condition that the network loops are greater than or equal to 4 and the reliability is the same with the value when we employing the ERL scheme, we can prolong the network lifetime at least:

$$
\Omega=\frac{E_{l}^{e}}{E_{l}^{u, m_{1}}} \times 100 \%=\frac{\zeta_{l}^{e, r} e_{u}^{r}+\zeta_{l}^{e, t} e_{u}^{t}}{\zeta_{l}^{e, r} e_{u}^{r}+\zeta_{l}^{e, t} e_{u}^{t}-\vartheta_{u}^{t} e_{u}^{t}} \times 100 \%
$$

$\vartheta_{u}^{t}$ represents the amount of data that decreased.

Proof For a planar network, if the number of network loops less than 4.

In case of the number of network loops is 2, we reduce the nodes' redundancy level in the first loop by 1 , and increase the nodes' redundancy level in the second loop until the nodal reliability are nearly to 1 . Because the increasing of redundancy level is much larger than 1 , so the improvement of the nodes' redundancy level in the second loop will greatly increase the received data sizes for the nodes in the first loop, as a result, it increases the energy consumption of the nodes in the first loop and shorten the network lifetime.

In case of the number of network loops is 3 , we reduce the redundancy level in the first loop by 1 , and increase

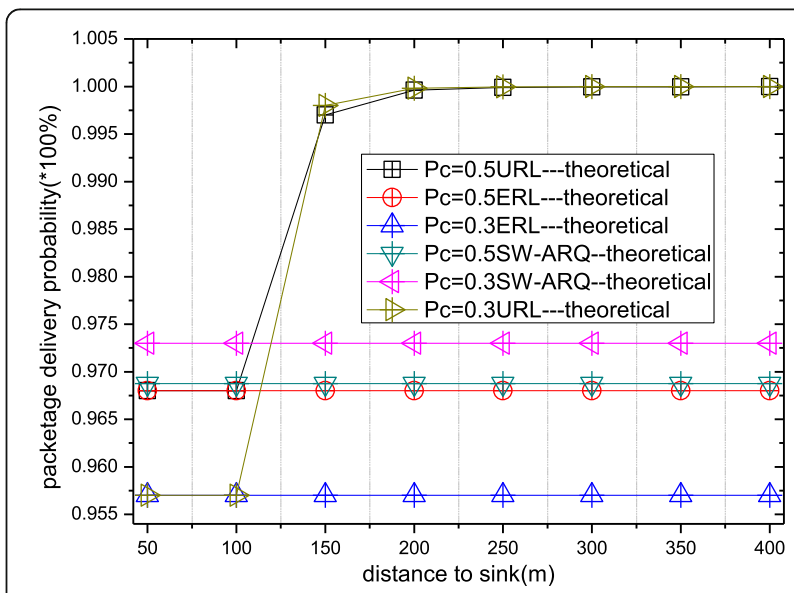

Fig. 14 Packet delivery probability for different mechanism under different link loss rates and different reliabilities 


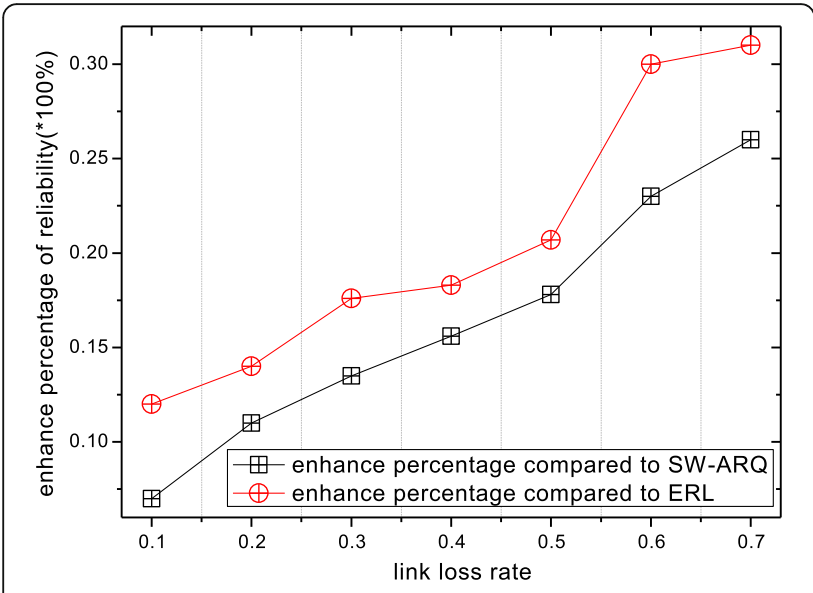

Fig. 15 The enhance percentage of the reliability for different link loss rate under same packet delivery probabilities $\left(p_{e}>70 \%\right)$

the nodes' redundancy level in the third loop until the reliability of the these nodes are nearly to 1 . Because of the nodes' redundancy level in the second loop are unchanged, under this condition, we would found that the nodes' energy consumption in the second loop is higher than that in the first loop. In this way, the network lifetime is also reduced.

Therefore, theorem 2 is only set up under the condition that the number of network loops greater than or equal to 4 .

For any network that its loops greater than or equal to 4 , the nodes' redundancy level is $m$ when we employ the ERL scheme, if we adopt the URL scheme, the initial value of parameters are all same as the ERL scheme, and in premise of the network reliability is unchanged, we can reduce the nodes' redundancy level in the first loop at least by 1 and improve the nodes' redundancy level in the outside loop to make the reliability near to 1 , in the meanwhile, we should also let $m_{n}$ meet the condition that the nodes' energy consumption in the $(n-1)^{t h}$ loop less than the energy consumption of nodes in the first loop. Reasons are as follows.

By formula (8), we can get the nodal reliability when we employing the ERL scheme.

Then the whole network reliability can express as

$$
\begin{aligned}
\hat{p}_{e} & =p^{\hbar} \mid \hbar \text { is the maximum hops in the network } \\
& =\left(\sqrt{\left.1-p_{c}^{m+2}-(m+2) \cdot p_{c}^{m+1} \cdot\left(1-p_{c}\right)\right)}\right)^{\hbar}
\end{aligned}
$$

is the maximum hops in the network

Adopting the URL scheme, we decrease the nodes' redundancy level in the first loop by 1 , by this time the nodal reliability in the first loop can be expressed as:

$$
p_{1}=\sqrt{1-p_{c}^{m+1}-(m+1) \cdot p_{c}^{m} \cdot\left(1-p_{c}\right)}
$$

We improve the redundancy level for the nodes in the outside loop within the threshold value as much as possible, and mark the value is $m_{n}$, by this time the nodal reliability in the outside loop can be expressed as

$$
p_{n}=\sqrt{1-p_{c}^{m_{n}+2}-\left(m_{n}+2\right) \cdot p_{c}^{m_{n}+1} \cdot\left(1-p_{c}\right)}
$$

The reliability of the intermediate link point does not change, still express as before: $m_{2}=m_{3}=\cdots=m_{n-1}=m$ and the reliability of them still express as $p_{2}=p_{3}=\cdots=p_{n-1}=p$.

By this time, the network reliability under the URL scheme can be expressed as

$$
\begin{aligned}
& \hat{p}_{u}= p_{1} p_{2} p_{3} \cdots p_{n}=p_{1} p^{h-2} p_{n} \approx \sqrt{1-p_{c}^{m+1}-(m+1) \cdot p_{c}^{m} \cdot\left(1-p_{c}\right)} \\
&\left(\sqrt{1-p_{c}^{m+2}-(m+2) \cdot p_{c}^{m+1} \cdot\left(1-p_{c}\right)}\right)^{h-2} \\
& \hat{p}_{u}-\hat{p}_{e}= \sqrt{1-p_{c}^{m+1}-(m+1) \cdot p_{c}^{m} \cdot\left(1-p_{c}\right)} \\
&\left.\left(\sqrt{1-p_{c}^{m+2}-(m+2) \cdot p_{c}^{m+1} \cdot\left(1-p_{c}\right.}\right)\right)^{h-2} \\
&-\left(\sqrt{1-p_{c}^{m+2}-(m+2) \cdot p_{c}^{m+1} \cdot\left(1-p_{c}\right)}\right)^{h}
\end{aligned}
$$

Obviously,

$$
\hat{p}_{u}-\hat{p}_{e} \geq 0
$$

Because of high energy consumption for the nodes in the inner loop, so when the energy is completely consume in the inner loop, we consider that the whole network is dead. Through above proof, we have known that we can reduce the redundancy level for the nodes in the first loop at least by 1 to reduce theirs energy consumption, prolong the network lifetime. At this time the decrease of the end-to-end reliability can be compensated by the increase of redundancy level for the nodes in the outer loops.

By formulas (18) and (19), we can calculate the amount of data that the nodes in the inner loops need to send and receive. If the node's redundancy level is reduced by 1 , though the receiving data sizes is unchanged, the sending data sizes is decreased,

$$
\begin{gathered}
\vartheta_{u}^{t}=\left(\frac{l+r}{l} \cdot p+\frac{l+2 r}{l} \cdot p^{2}+\cdots+\frac{l+z r}{l} \cdot p^{z-1}+2\right) \\
\lambda \mid l=h r+x, x+z r \leq R
\end{gathered}
$$

Accordingly, the saved energy can be expressed as

$$
E_{\vartheta}^{u}=\vartheta_{u}^{t} e_{u}^{t}
$$

By formula (16), the node's energy consumption under ERL scheme is $E_{l}^{e}=\zeta_{l}^{e, r} e_{u}^{r}+\zeta_{l}^{e, t} e_{u}^{t}$ 
The node's energy consumption under URL scheme in the same condition can be expressed as

$$
E_{l}^{u, m_{1}}=\zeta_{l}^{e, r} e_{u}^{r}+\zeta_{l}^{e, t} e_{u}^{t}-\vartheta_{u}^{t} e_{u}^{t}
$$

So, adopting URL scheme, the network lifetime can enhance at least:

$$
\Omega=\frac{E_{l}^{e}}{E_{l}^{u, m_{1}}} \times 100 \%=\frac{\zeta_{l}^{e, r} e_{u}^{r}+\zeta_{l}^{e, t} e_{u}^{t}}{\zeta_{l}^{e, r} e_{u}^{r}+\zeta_{l}^{e, t} e_{u}^{t}-\vartheta_{u}^{t} e_{u}^{t}} \times 100 \%
$$

On the basis of theorem 2, Fig. 16 presents under the condition that in different link qualities $\left(p_{c}=0.3\right.$ and $p_{c}$ $=0.5$ ), and ensure the end-to-end reliability greater than or equal to $70 \%$, when reducing the redundancy level for the nodes in hotspot areas by 1 , the nodes' redundancy level in different areas yet. From Fig. 16, when we decrease the redundancy level for the nodes in the inner loop by 1 , the rest energy of nodes in the farthest area can improve their redundancy level to $m=50$ in order to meet the condition that end-to-end reliability $p_{e} \geq 70 \%$, at this time, the hop-to-hop reliability in different areas are shown in Figs. 17, 18, and 19 present that under the different link qualities $\left(p_{c}=0.3\right.$ and $\left.p_{c}=0.5\right)$, meeting the condition that end-to-end reliability greater than or equal to $70 \%$, the energy consumption for the nodes in different loops. It is observed from Figs. 18 and 19 that after decreasing the nodes' redundancy level in the first loop, we could guarantee $p_{e} \geq 70 \%$ only by improving the nodes' redundancy level in the farthest area, while there are still many regional nodes remaining energy in the network by now. The relevant parameters of SW-ARQ approach are all presented in Table 4.

Actually, it is likely to continue to decrease the nodes' redundancy level in the first loop and promote the nodes' redundancy level in the non-hotspot areas, then further

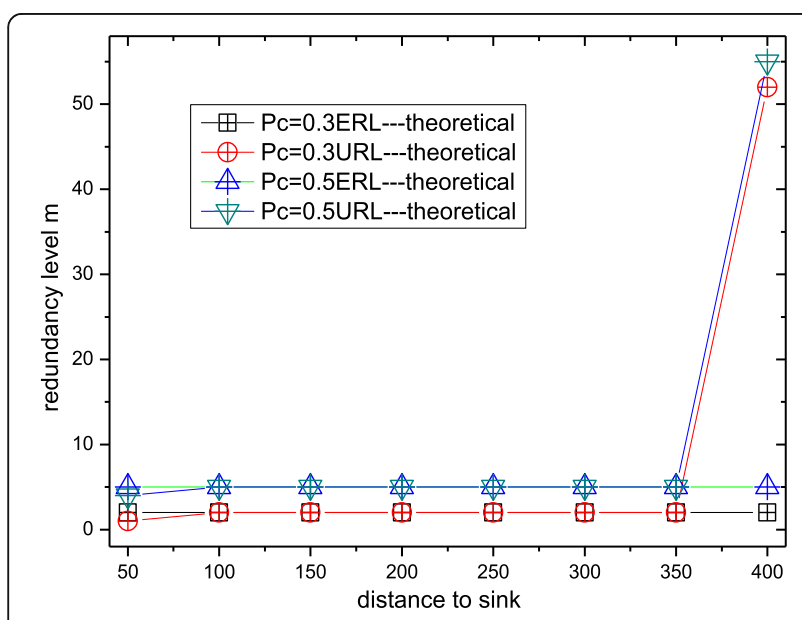

Fig. 16 Redundancy level for different mechanisms

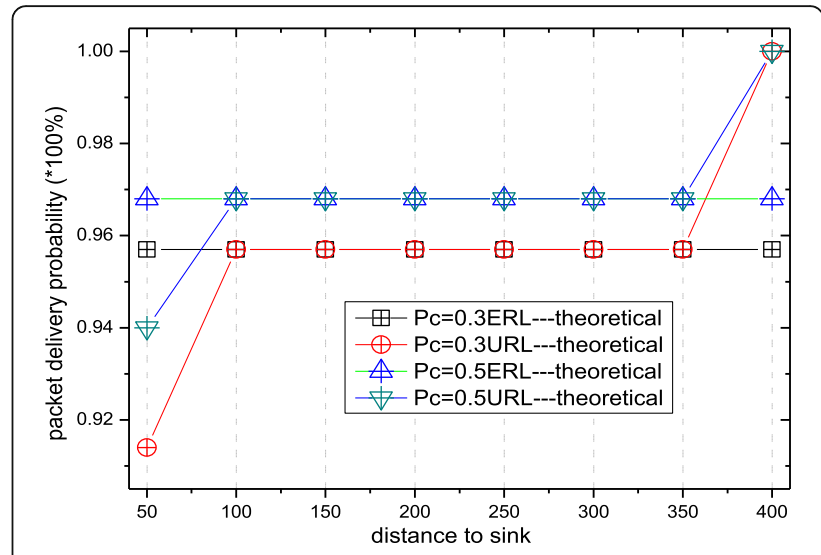

Fig. 17 Redundancy level for different mechanisms

improving the network lifetime. This suggests that theorem 2 is given the lower bound that the URL scheme could improve the network lifetime in premise of ensuring the equal end-to-end reliability with ERL and SW-ARQ approach.

Figure 20 presents under the different link qualities, the that ratio of the URL scheme can enhance the lifetime contrast with ERL scheme and SW-ARQ scheme. From the experimental results, the lifetime under the the URL scheme is 1.05-1.44 times to the network that under the ERL scheme and it is $0.61-0.89$ times to the network that under SW-ARQ approach. Figure 21 shows under the different reliability, the ratio of the URL scheme can enhance the lifetime contrast with ERL scheme and SW-ARQ approach. From the experimental result in Fig. 21, when the link qualities are 0.2, 0.3, and 0.5 , the lifetime under the URL scheme, respectively are 1.14-1.25 times, $1.18-1.44$ times, $1.08-1.13$ times to the network that under ERL scheme, and it is 1.003-1.15 times, 0.96-1.08 times, and 0.82-0.97 times to the network that are under thr SW-ARQ approach.

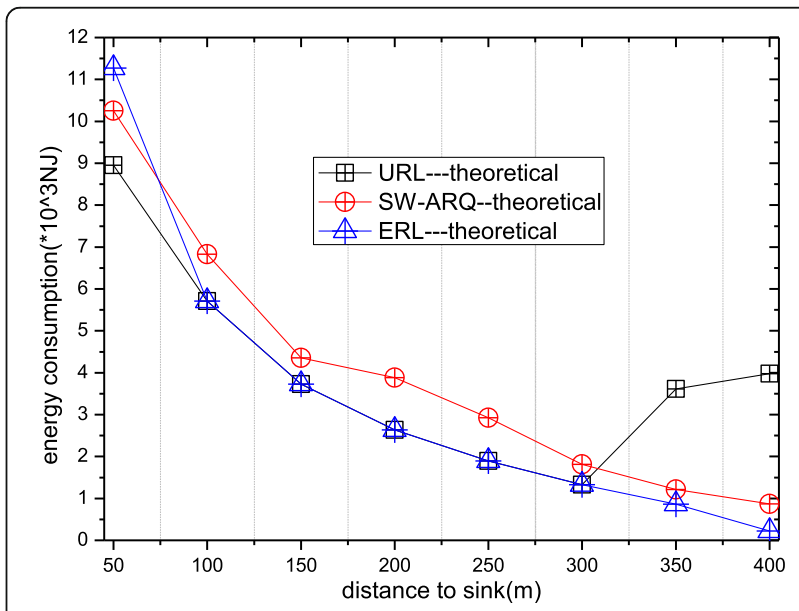

Fig. 18 Energy consumption for different mechanisms $\left(p_{c}=0.3\right.$, $\left.p_{e} \geq 70 \%\right)$ 


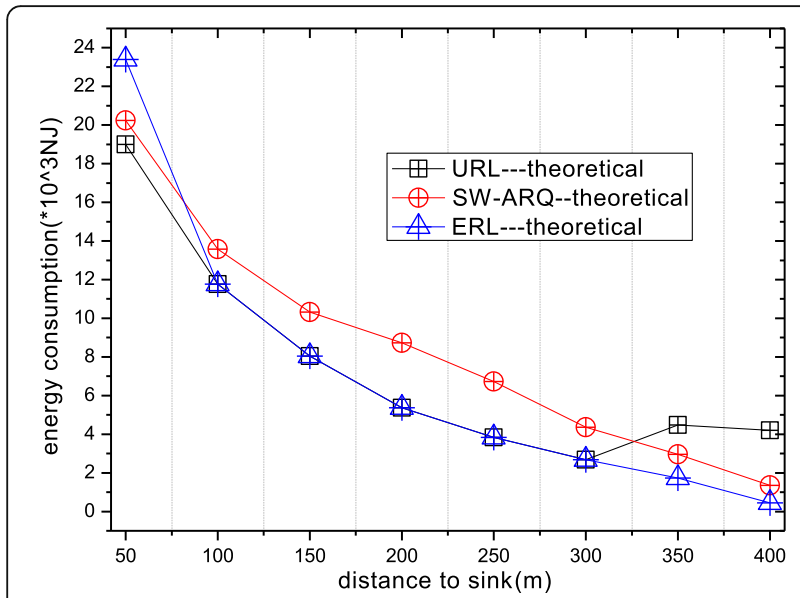

Fig. 19 Energy consumption for different mechanisms $\left(p_{c}=0.5\right.$, $\left.p_{e} \geq 70 \%\right)$

\subsection{Delay analysis}

Theorem 3 Under the same reliability $p_{e}$, the delay under the URL scheme can be expressed as

$$
R_{D_{\text {URL }}}=\sum_{i=1}^{n}\left(T\left(\frac{1}{R_{P m_{i}}}-1\right)\right)
$$

In the formula, $R_{D_{U R L}}$ represents the delay caused by employing URL scheme, $R_{P m_{i}}$ is the packet pair reception for the $i^{\text {th }}$ node, $\mathrm{T}$ refers to the retransmission time interval.

Proof According to the ref. [8], in the ERL scheme, the delay is present as

$$
R_{D_{E R L}}=\left(T_{X}-1\right) H \hbar=\left(\frac{1}{R_{P m}}-1\right) H \hbar
$$

$H$ represents the number of hops to base station, $T_{X}$ denotes the expected transmission count, $\hbar$ is the number of hops to the base station, $\mathrm{T}$ is the retransmission

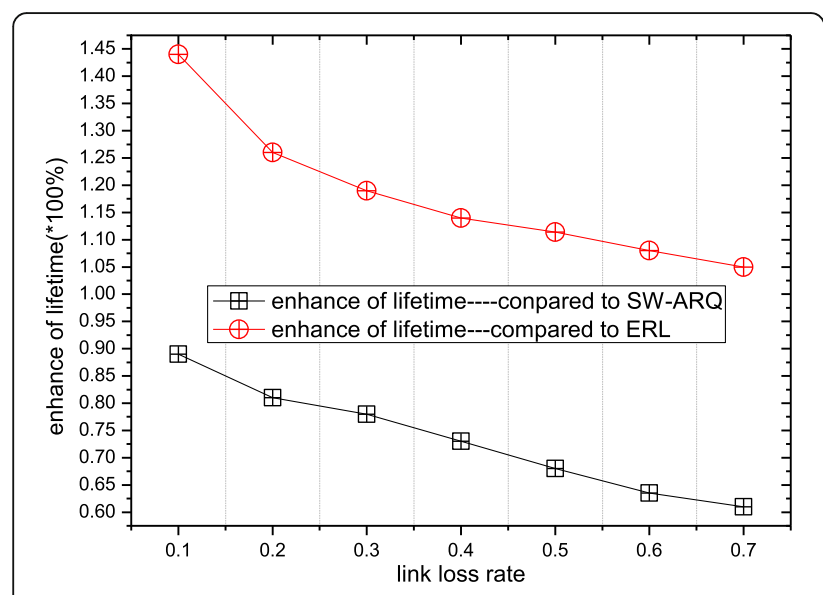

Fig. 20 The improve of lifetime for different link qualities

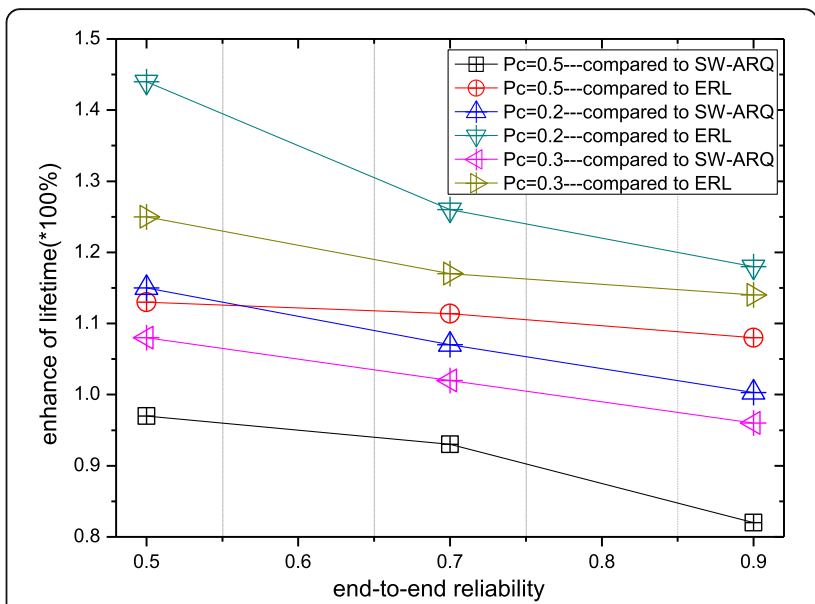

Fig. 21 The improve of lifetime for different link qualities and reliabilities

time interval, $R_{P m}$ is when we use $m$ redundant packets for each pair, the packet pair reception probability, that is

$$
\begin{aligned}
R_{P m}= & 1-\left(\begin{array}{c}
m+2 \\
0
\end{array}\right)\left(1-p_{q}\right)^{m+2}-\left(\begin{array}{c}
m+2 \\
1
\end{array}\right) \\
& \times\left(1-p_{q}\right)^{m+1} \cdot p_{q} \mid p_{q}=1-p_{l}
\end{aligned}
$$

In URL scheme, there are different redundancy levels $m_{1}, m_{2}, m_{3}, \cdots, m_{n}$ for each hop, according to type (42), we can get the packet pair reception $R_{P m_{1}}, R_{P m_{2}}, R_{P m_{3}}, \cdots$ , $R_{P m_{n}}$, then we can get the delay for the $i^{\text {th }}$ hop:

$$
R_{D i}=\left(\frac{1}{R_{P m_{i}}}-1\right) \hbar
$$

Therefore, it can be concluded that the retransmission delay under URL approach

$$
R_{D_{\text {URL }}}=\sum_{i=1}^{n}\left(T\left(\frac{1}{R_{P m_{i}}}-1\right)\right)
$$

The test scenarios of this section are in a linear network that 10 nodes array in a straight line. Figure 22 shows the contrast figure of delay between three different schemes under different link qualities, in premise of guaranteeing the end-to-end reliability greater than or equal to $70 \%$. Figure 23 presents the redundancy level for each node. It can be observed that in premise of ensuring a high lifetime of network and the end-to-end reliability greater than $70 \%$, the delay of SW-ARQ approach is still much longer than that of the other two methods, and the delay incurred by URL scheme is higher than the ERL scheme incurred, but with the increase of link loss rate, the difference of delay under two methods gradually narrowed, finally remaining at around $9 \%$. While the less delay increased is insignificant for 


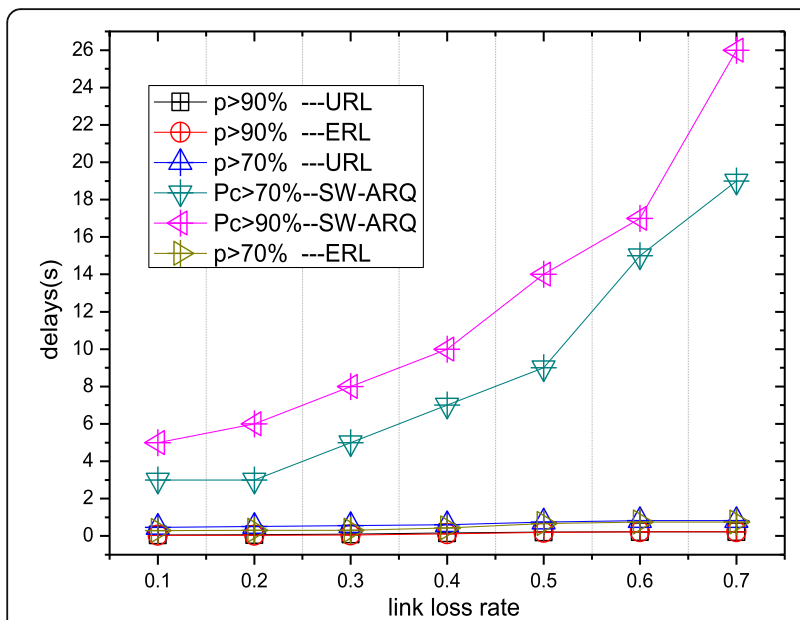

Fig. 22 Delay for different mechanisms under same reliability $\left(p_{e}>70 \%\right)$

the improvement of the reliability and lifetime network performance. And with the improvement of the reliability, the difference of delay under ERL and URL methods also gradually narrowed, when the reliability greater than $90 \%$, the delays are almost equal, by this time, the proposed method have a great improvement on reliability and lifetime performance. Especially aiming at the delayinsensitive application, the URL scheme has great advantage.

\section{Experimental results}

In order to evaluate the URL scheme performance in large scale sensor networks, we use Omnet++ to generate larger scenarios [25]. In this simulation, if there are no special instructions, the experimental scenarios are as follows: one sink node and 1000 sensor nodes are randomly deployed in a circular network, which the radius

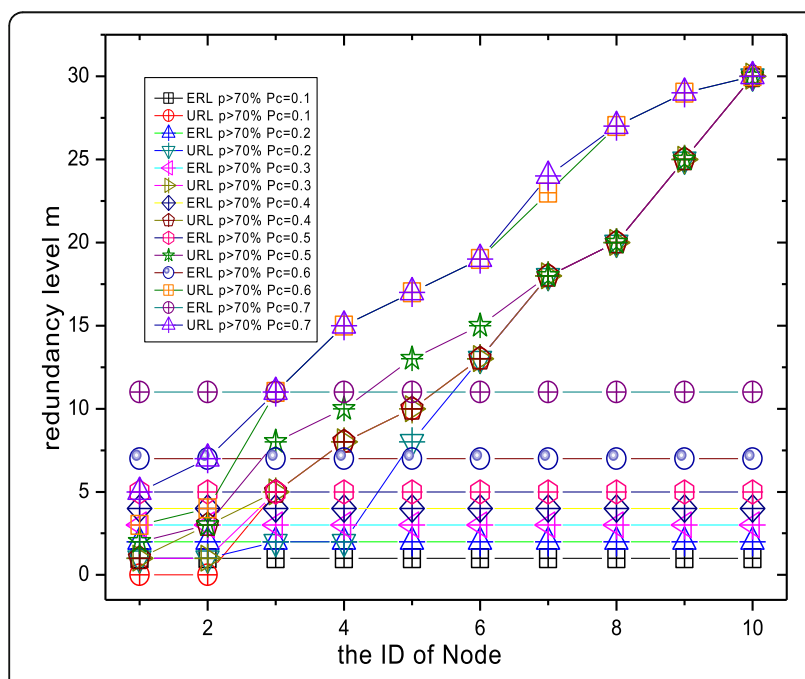

Fig. 23 Redundancy level under same reliability $\left(p_{e}>70 \%\right)$

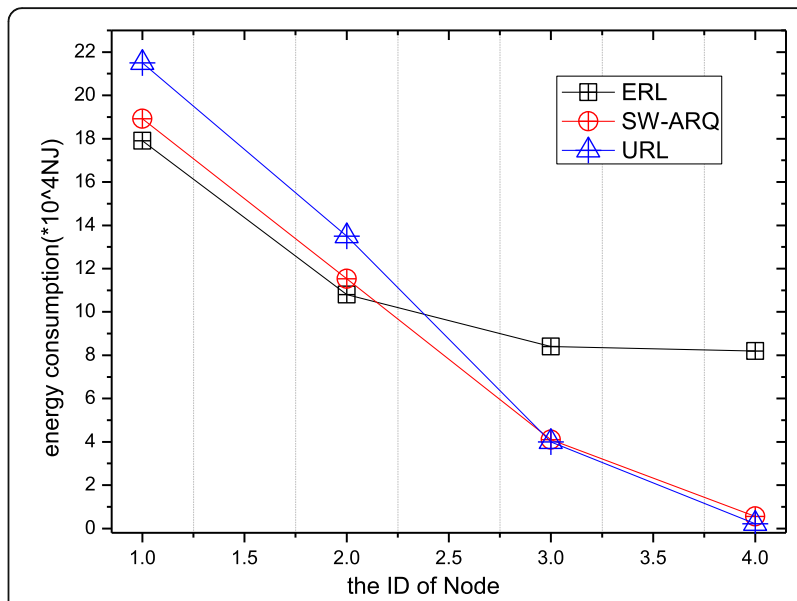

Fig. 24 Energy consumption for different mechanisms (4 hops)

is $400 \mathrm{~m}$ and the nodal transmission radius is $50 \mathrm{~m}$. The routes were selected based on the shortest routing algorithm [22]. Other parameters are the same as those in section 3. For SW-ARQ approach, the relevant parameters are listed in Table 4.

\subsection{Energy efficiency}

The experimental scenario of Figs. 24, 25, and 26 is in the linear network, that is all nodes in the network deployment on a straight line, the base station locate on the one end of this line. Figures 24, 25, and 26 are given, respectively, that when the number of nodes are 4,7 , and 10, and in premise of ensuring the same end-to-end reliability $\left(p_{e}>70 \%\right)$, the comparison of nodes' energy consumption under different methods. The experimental results are consistent with the theoretical analysis above. Because the URL scheme decrease the nodes' redundancy level in hot-spot area and increase the nodes' redundancy level in non-hotspot area, so the energy consumption characteristics are the nodes' energy

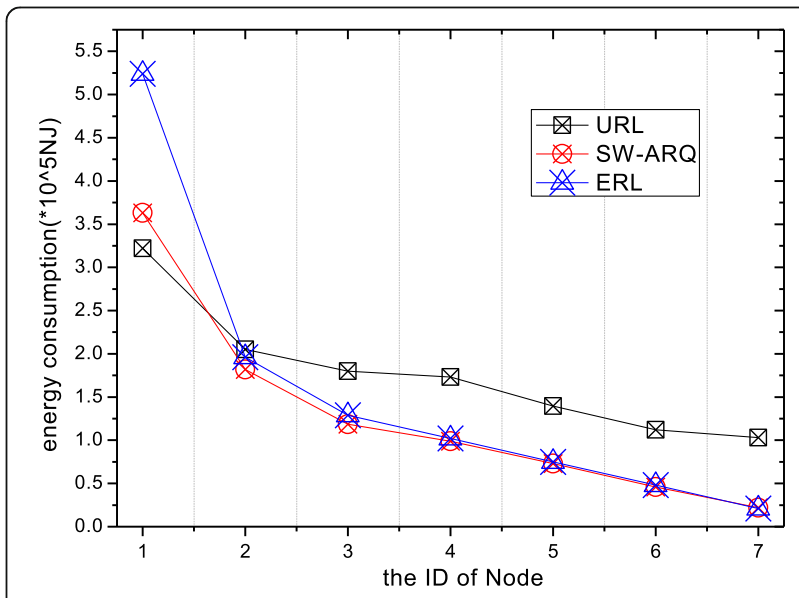

Fig. 25 Energy consumption for different mechanisms (7 hops) 


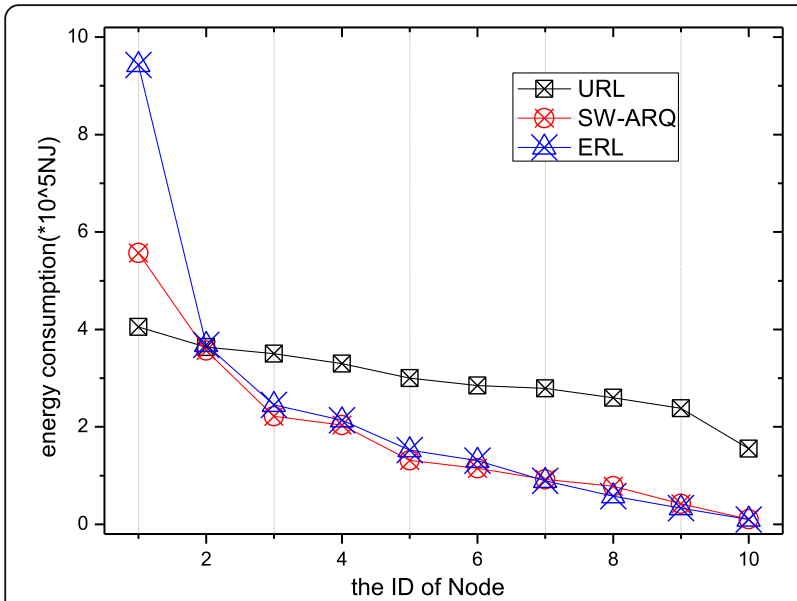

Fig. 26 Energy consumption for different mechanisms (10 hops)

consumption in near to sink areas under URL approach is lower than that under ERL approach and SW-ARQ approach, and the nodes' energy consumption in far to sink areas under URL approach is higher than that under ERL and SW-ARQ. Because the lifetime is depended on the largest energy consumption node, so the approach proposed in this paper could prolong the network lifetime efficiently.

The experimental results of Figs. 27, 28, 29, and 30 are under the experimental scenario that one sink node and 1000 sensor nodes were randomly deployed in an area of disk network, the radius of this network is $400 \mathrm{~m}$ and the nodal transmission radius is $50 \mathrm{~m}$. Figure 27 presents the energy consumption under the same link loss rate and different reliability. Figure 28 shows the energy consumption with two approaches under different link qualities, in premise of ensuring the end-to-end reliability greater than $70 \%$. Figure 29 presents the nodes' redundancy level with two approaches under the same

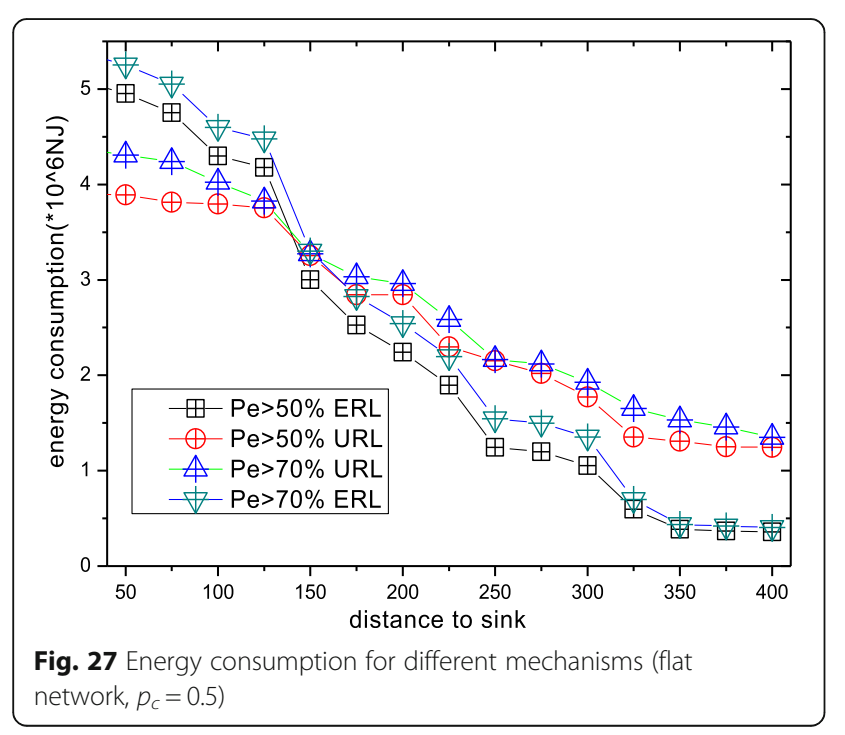

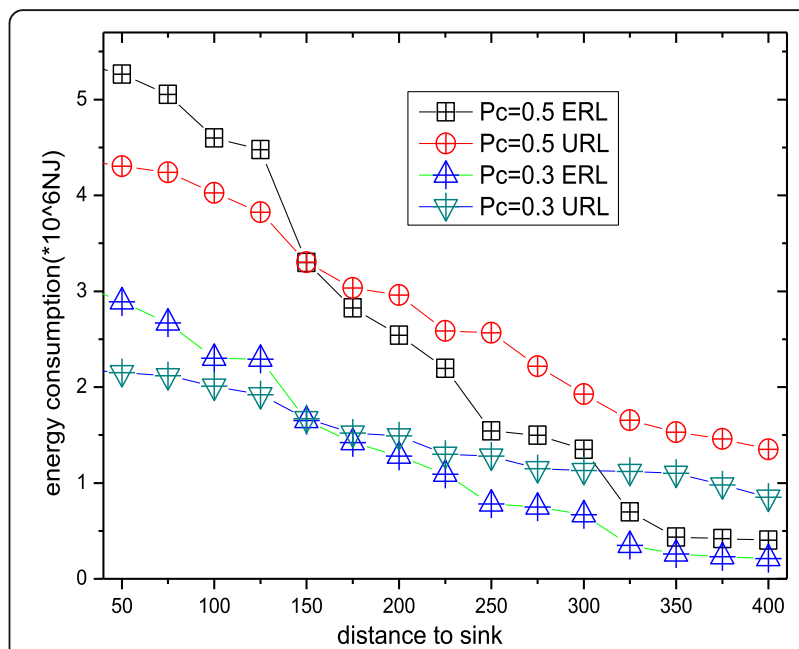

Fig. 28 Energy consumption for different mechanisms (flat network, $\left.p_{e} \geq 70 \%\right)$

link loss rate and different reliability. Figure 30 shows the nodes' redundancy level with two approaches under same reliability and different link loss rate. The experimental results are all consistent with the theoretical analysis. For specifics: (1) URL scheme has lower energy consumption for the nodes in the regions near to sink and higher energy consumption for the nodes in the regions far to sink. (2) The URL scheme has lower redundancy level for the nodes in the regions near to sink and higher redundancy level for the nodes in the regions far to sink.

Figure 31 presents the nodes' energy consumption when adopting the URL scheme under the condition that $p_{c}=0.5, p_{e}>70 \%$. Comparing with Fig. 3 (the energy consumption of ERL scheme), URL scheme has a much more balanced energy consumption than ERL

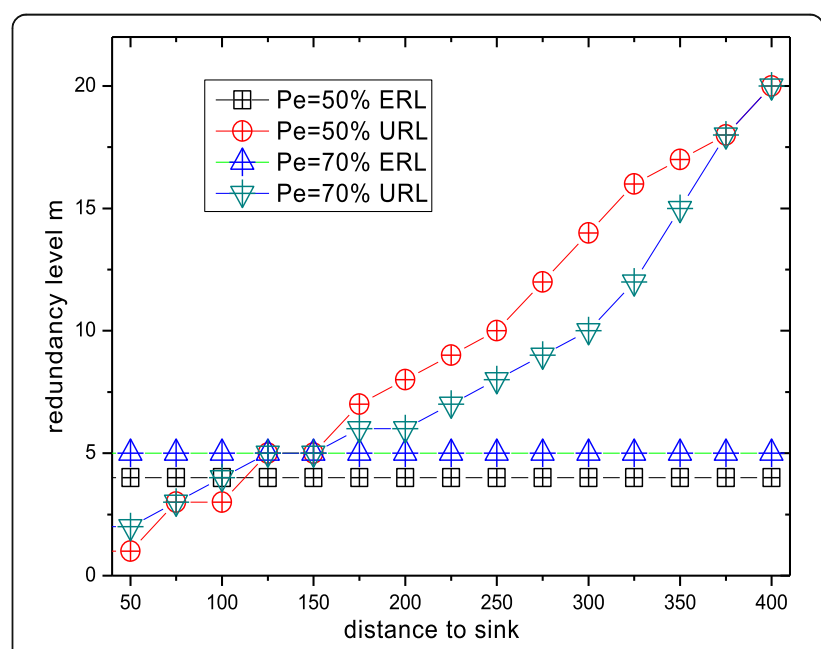

Fig. 29 Redundancy level under different reliabilities (flat network, $p_{C}=0.5$ ) 


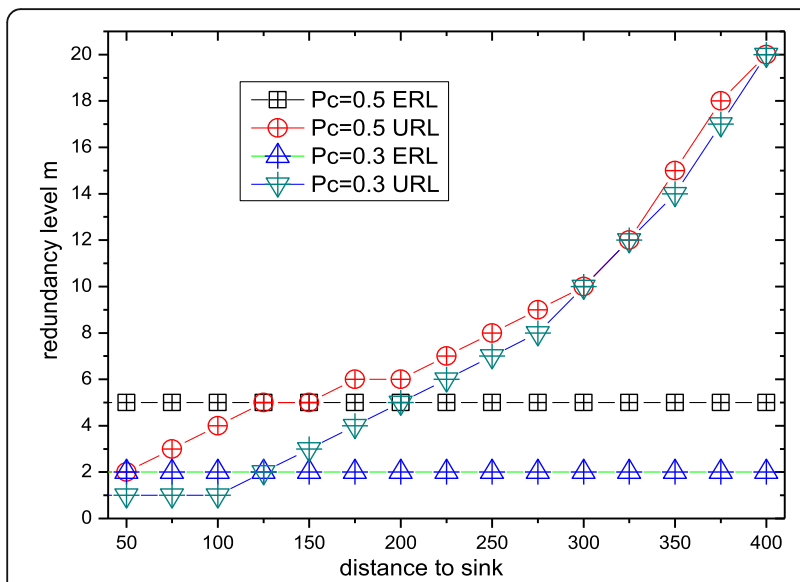

Fig. 30 Redundancy level under different reliabilities (flat network, $\left.p_{e}>70 \%\right)$

scheme and as the network die, the remaining energy is less than $15 \%$ in the whole network, which suggested that URL scheme has a higher energy efficiency and has a better performance than other strategies.

\subsection{Lifetime of the network}

Figure 32 presents the experimental results by contrasting the network lifetime with different methods under the different scales of linear network. From the experimental results, in premise of ensuring the same end-to-end reliability, the URL scheme could improve the lifetime by $15-50 \%$ than ERL approach and it also could improve the lifetime by $7-30 \%$ than SW-ARQ approach. Figure 33 presents the experimental results by contrasting the network lifetime under the different radiuses of planar network. The results show as well, relative to ERL scheme and SW-ARQ approach, URL scheme has excellent performance. Comparing with ERL scheme, it could improve the lifetime by $15-23.4 \%$; comparing with SW-ARQ approach, it could improve

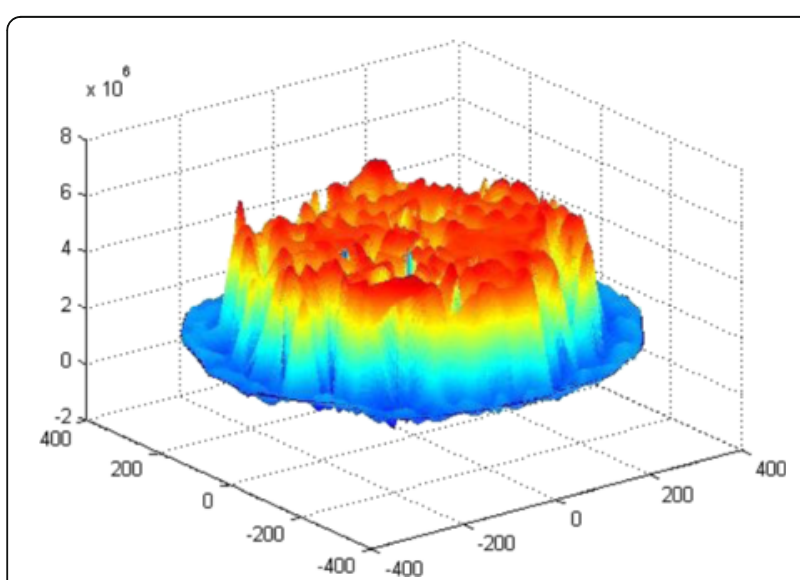

Fig. 31 The energy consumption with unequal redundancy level $\left(p_{C}=0.5, p_{e}>70 \%\right)$

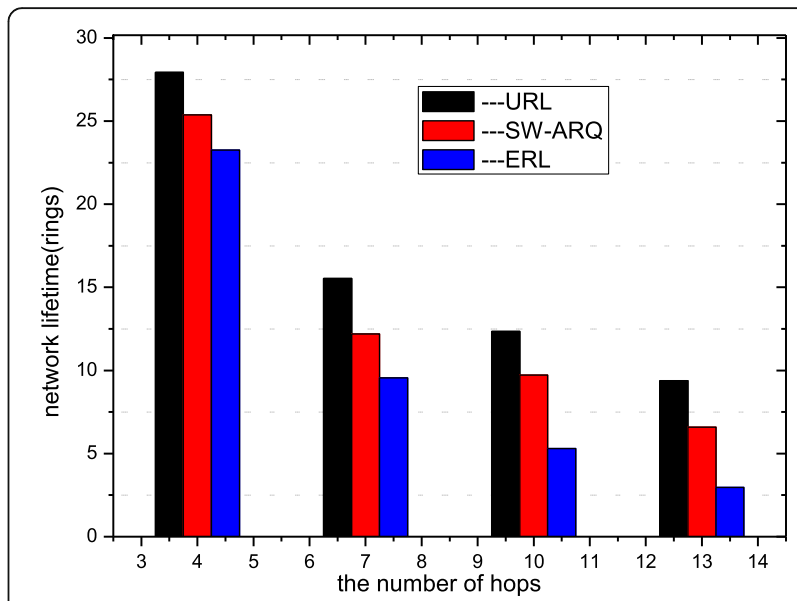

Fig. 32 lifetime contrasting under different scales linear networks $\left(p_{e}>70 \%, p_{c}=0.5, E_{i n i}=5^{*}(10 \wedge 6) \mathrm{NJ}\right)$

the lifetime by $6-16 \%$. Figures 34 and 35 show the experimental results by contrasting the network lifetime with URL scheme and ERL scheme under the different link loss rate and different reliability. URL scheme can respectively improve the lifetime by $20-44.4 \%$ and 12.1-30\%. This shows that the proposed URL strategy has a much higher lifetime than the ERL strategy under different network scenarios and different parameters, so the URL scheme has a satisfactory performance.

\subsection{Reliability}

Figure 36 shows the experimental results by contrasting the reliability with different methods in premise of ensuring the same lifetime under the different scales of linear network. From the experimental results, comparing with ERL scheme, the proposed strategy in this paper can improve the network reliability by $14.1-28.3 \%$; comparing with SW-ARQ approach, the proposed

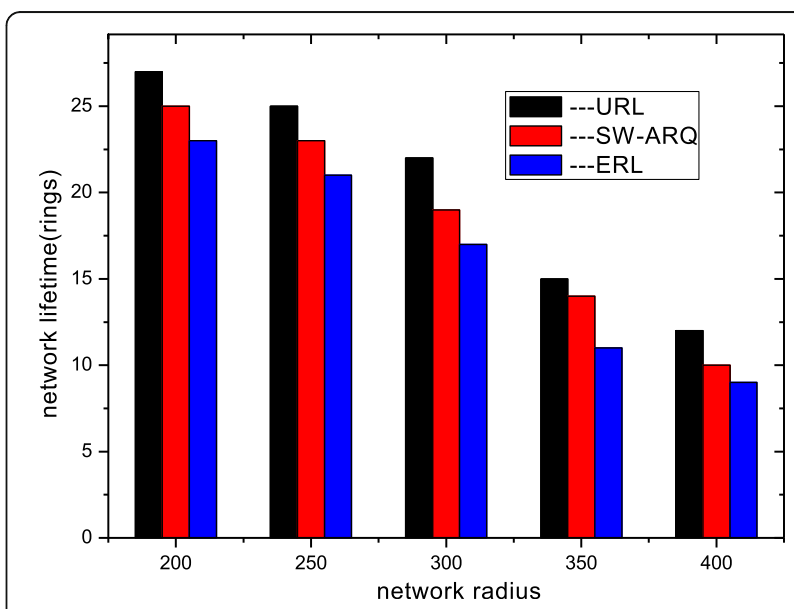

Fig. 33 lifetime contrasting under different scales planar networks $\left(p_{e}>70 \%, p_{c}=0.5, E_{\text {ini }}=5^{*}(10 \wedge 7) \mathrm{NJ}\right)$ 


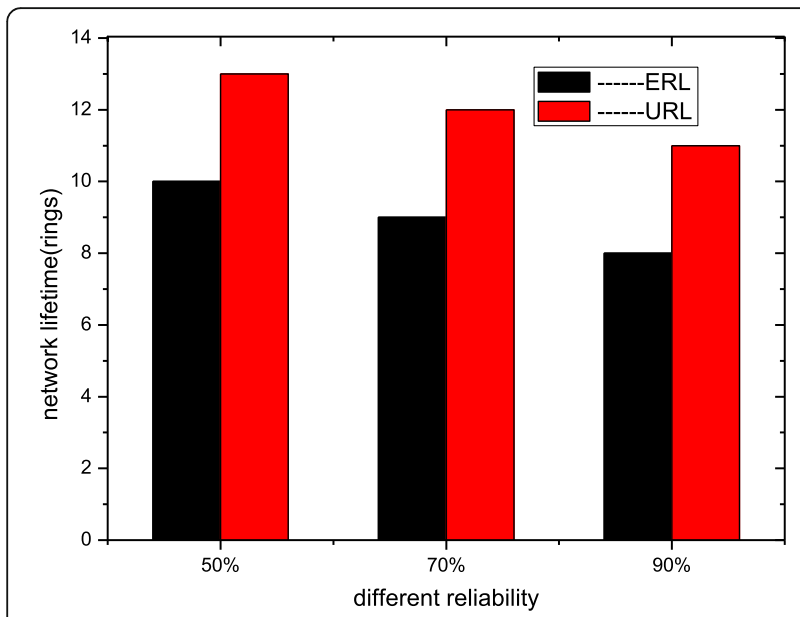

Fig. 34 lifetime contrasting under same link loss rate and different $p_{e}\left(p_{c}=0.5, E_{\text {ini }}=5^{*}(10 \wedge 7) \mathrm{NJ}\right)$

strategy in this paper can improve the network reliability by $8-23 \%$. Fig. 37 presents the experimental results by contrasting the network reliability under the different radiuses of planar network. Relative to ERL scheme, the URL scheme can improve the reliability by $14.5-23.6 \%$. And relative to SW-ARQ approach, it can improve the reliability by $10-15 \%$. Figure 38 shows the results by contrasting the reliability with ERL and URL scheme under the same lifetime and different link loss rate. And from Fig. 39, the proposed URL strategy has higher network reliability than that of the ERL strategy under different network scenarios and different parameters, so the URL scheme has a satisfactory performance on the reliability.

\subsection{The delay}

Relative to the theoretical analysis results in 5.3, the experimental results will be given in this section. Figure 40

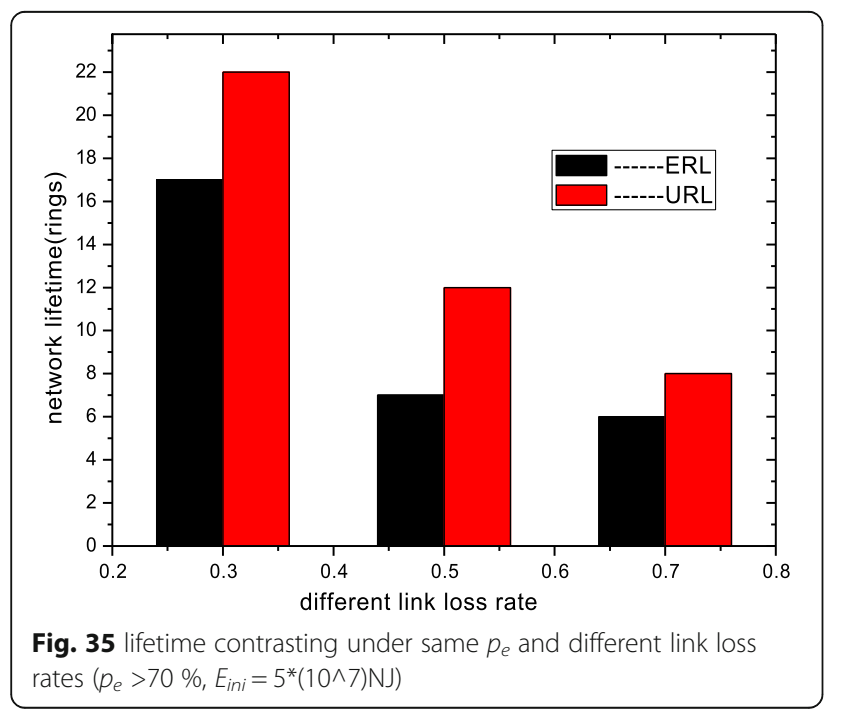

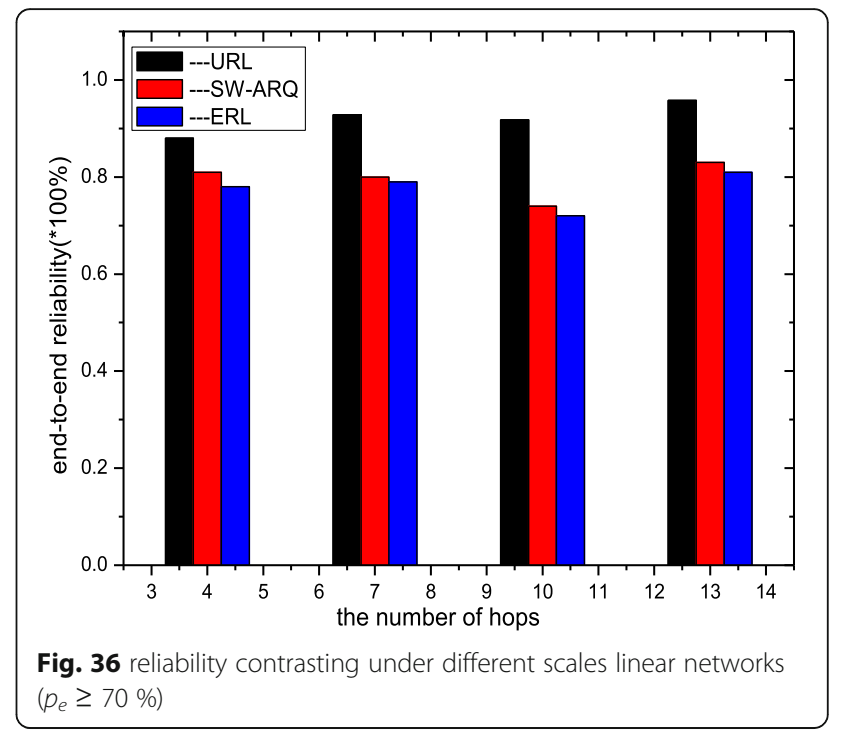

shows the delay contrasted with different methods under the same end-to-end reliability and different link qualities. It can be observed from the picture that: the delay of SW-ARQ approach is much larger than that of the other two methods, and when the link loss rate is small, the delay incurred by the URL strategy is longer than the delay incurred by ERL strategy under the same reliability. With the increase of the link loss rate, the gap gradually narrowed, when the link loss rate greater than 0.6 , the difference of delay incurred by two methods almost remaining at around $9 \%$ unchanged. From the experimental results, we can draw the conclusion that (1) the experimental results are consistent with the above theoretical analysis. (2) Although the URL strategy may have a disadvantage on the delay performance, under the same condition, the URL approach can prolong the

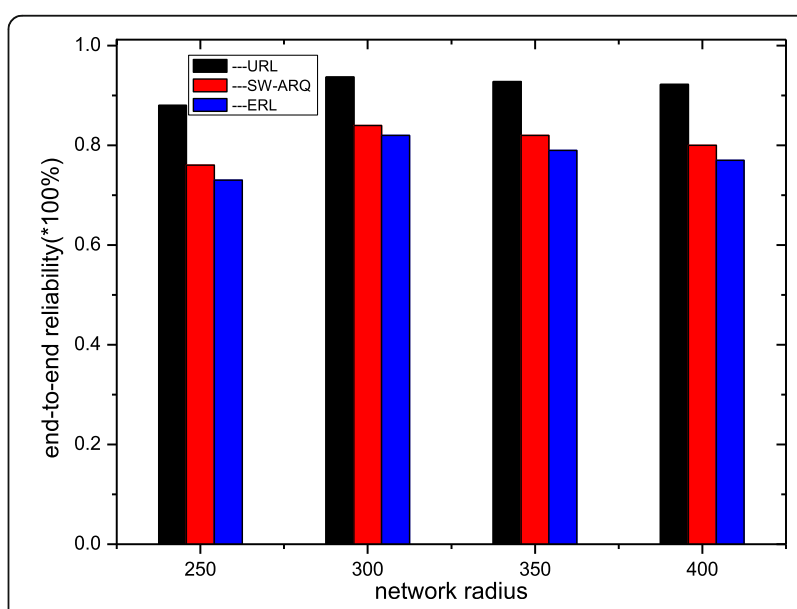

Fig. 37 reliability contrasting under different scales planar networks $\left(p_{e} \geq 70 \%\right)$ 


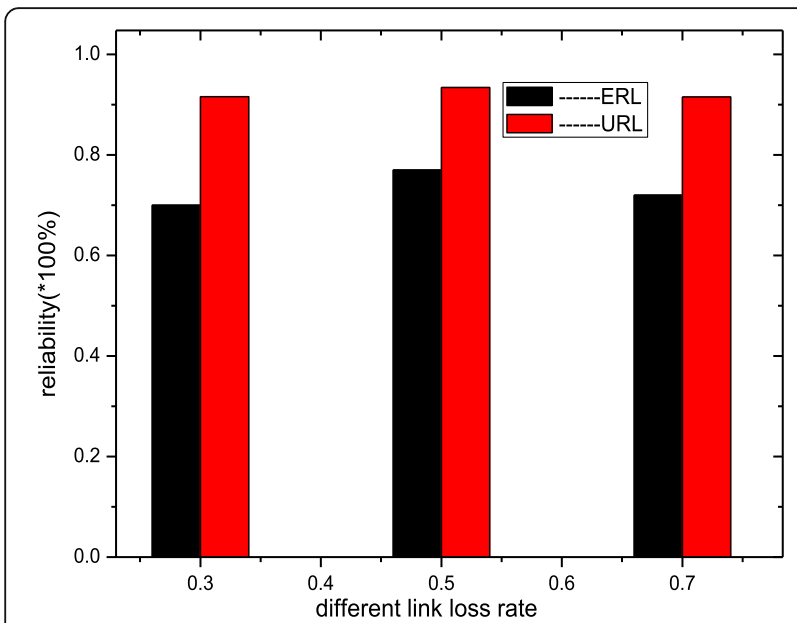

Fig. 38 Reliability contrasting under different link loss rate $\left(p_{e} \geq 70 \%\right.$, network radius $R=400$, transmit radius $r=50$ )

lifetime $20-44.4 \%$ by analyzing in 6.2 . So, it still has a great advantage and a better performance.

Figure 41 presents the delay contrasted with ERL and URL approaches under the same lifetime. We can see the delay differential become narrowing with the increase of the reliability or the increase of the link loss rate. When the reliability is greater than $90 \%$, the delays incurred by two methods are nearly equal. From the 6.3, we can see that the URL scheme can improve the reliability by $14.5-23.6 \%$, which suggesting that URL scheme is better.

\section{Conclusions}

In the wireless network where the energy is limited and the link loss rate is big, it is a challenge issue to guarantee the end-to-end reliability and high lifetime at the same time. We proposed a network coding assisted

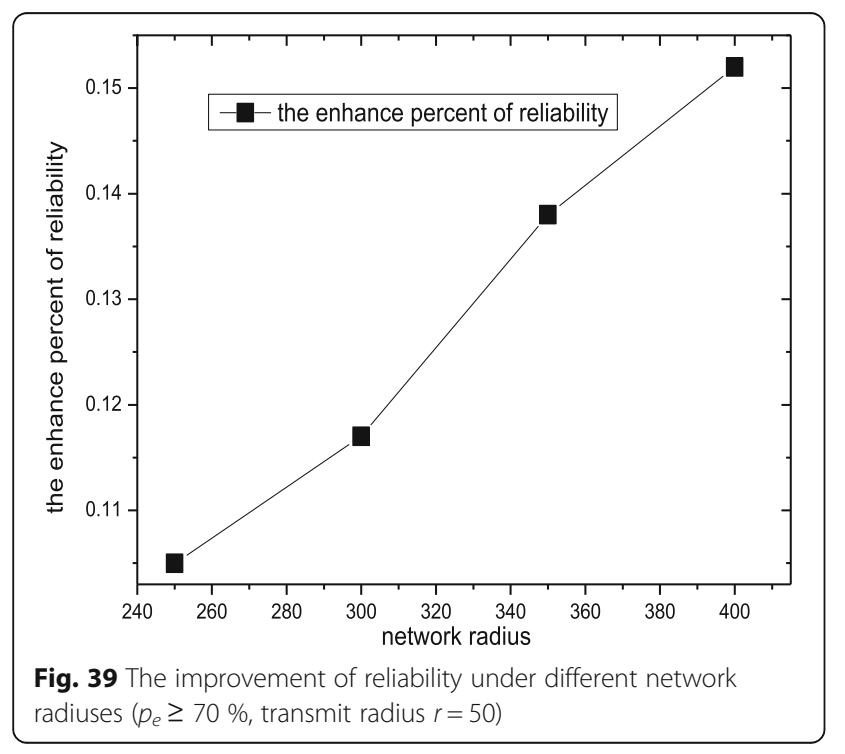

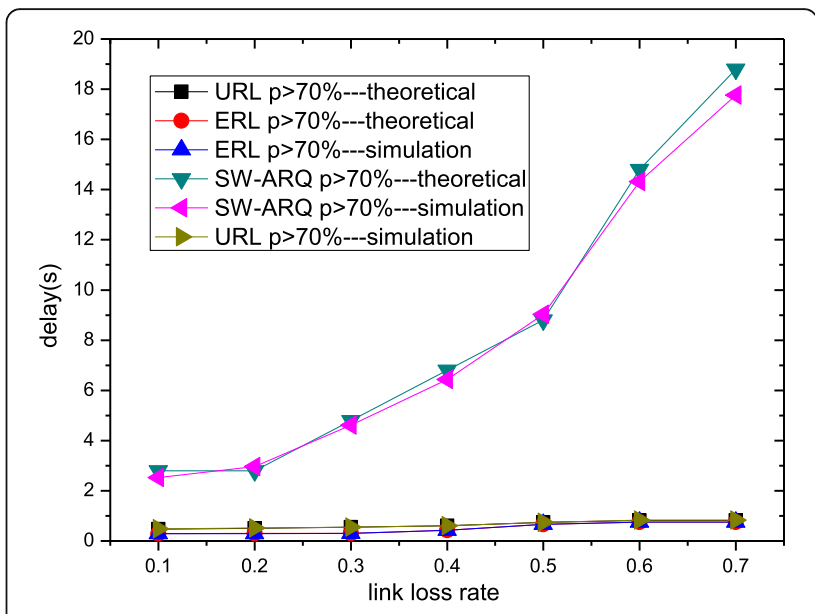

Fig. $\mathbf{4 0}$ Delay under different link qualities with same reliability $\left(p_{e}>70 \%\right)$

unequal redundancy level based mechanism for wireless sensor networks. The proposed scheme can attain a higher end-to-end network reliability, and longer network lifetime with high energy efficiency. What is more, the URL scheme can be easily adopted by vary network coding based data collection scheme and provides an easily implementable solution for reliable and high lifetime data collection in WSNs. We use Omnet++ network simulator, and theoretical analysis to evaluate the performance of the proposed scheme. The evaluation results indicate that: relative to ERL approach, under the same reliability, the URL scheme can improve the network lifetime by $15-50 \%$; and under the same lifetime, the URL scheme can improve the reliability by 14.1-28.3\%. In large-scale sensor networks, the delay incurred by two different methods is nearly equal. Relative to SW-ARQ approach, under the same reliability, the URL scheme can improve the network lifetime by

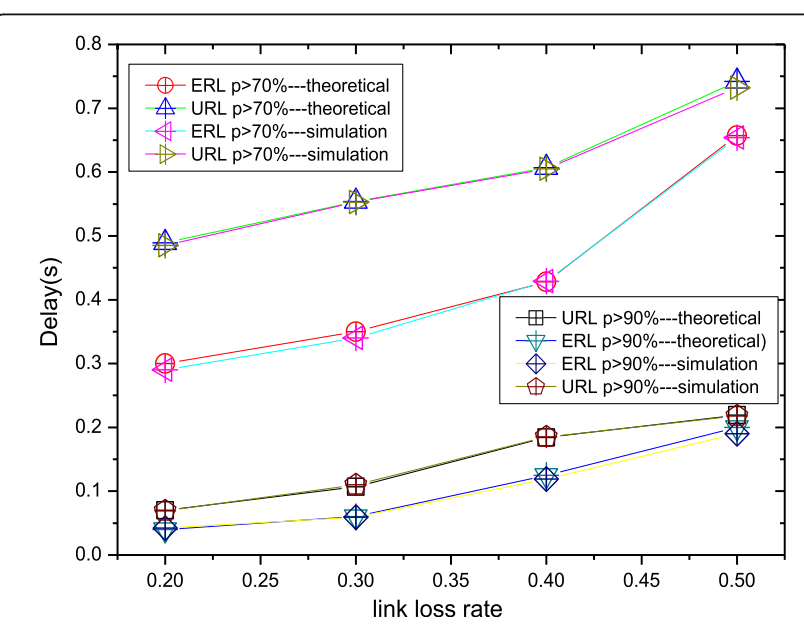

Fig. 41 Delay under different link qualities and different reliabilities with same lifetime 
7-30\%; and under the same lifetime, the URL scheme can improve the reliability by $8-23 \%$. The delay of SW-ARQ approach is much larger than URL scheme incurred. These are all suggested that the URL scheme is an efficiency data collection scheme.

\section{Acknowledgements}

This work was supported in part by the National Natural Science Foundation of China (61379110, 61073104, 61272149, and 61272494), The National Basic Research Program of China (973 Program) (2014CB046305).

\section{Authors' information}

Qi Zhang received B.Sc on 2015. Currently she is a master in School of Information Science and Engineering of Central South University, China. Her research interest is wireless sensor network.

Anfeng Liu is a Professor of School of Information Science and Engineering of Central South University, China. He is also a member (E200012141M) of the China Computer Federation (CCF). He received his M.Sc. and Ph.D degrees from Central South University, China, 2002 and 2005, both in computer science. His major research interests are wireless sensor network and crowd sensing network.

\section{Competing interests}

The authors declare that they have no competing interests.

Received: 18 March 2016 Accepted: 8 October 2016

Published online: 26 October 2016

\section{References}

1. S He, J Chen, X Li et al., Mobility and intruder prior information improving the barrier coverage of sparse sensor networks. IEEE Trans. Mob. Comput. 13(6), 1268-1282 (2015)

2. Y Liu, M Dong, K Ota, A Liu, ActiveTrust: secure and trustable routing in wireless sensor networks. IEEE Trans. Inf. Forensics Secur. 11(9), 2013-2027 (2016)

3. JS Gui, M Ahmadi, F Tong, Dynamically constructing and maintaining virtual access points in a macro cell with selfish nodes. J. Syst. Softw. 108, 1-22 (2015)

4. $\mathrm{Y} \mathrm{Hu}, \mathrm{A}$ Liu, Improvement the quality of mobile target detection through portion of node with fully duty cycle in WSNs. Comput. Syst. Sci. Eng. 31(1), 5-17 (2016)

5. A Saifullah, Y Xu, C Lu et al., End-to-end communication delay analysis in industrial wireless networks. IEEE Trans. Comput. 64(5), 1361-1374 (2015)

6. A Liu, X Liu, Y Liu, A comprehensive analysis for fair probability marking based traceback approach in WSNs. Secur. Commun. Netw. 9(14), 2448-2475 (2016)

7. Q Yang, S He, J Li, J Chen, Y Sun, Energy-efficient probabilistic area coverage in wireless sensor. IEEE Trans. Veh. Technol. 61(1), 367-377 (2015)

8. C Wu, Y Ji, J Xu et al., Coded packets over lossy links: a redundancy-based mechanism for reliable and fast data collection in sensor networks[J]. Comput. Netw. 70, 179-191 (2014)

9. M Delamo, S Felici-Castell, JJ Perez-Solano, A Foter, Designing an open source maintenance-free environmental monitoring application for wireless sensor networks. J. Syst. Softw. 103, 238-247 (2015)

10. Z Rosberg, RP Liu, TL Dinh et al., Statistical reliability for energy efficient data transport in wireless sensor networks. Wirel. Netw. 16(7), 1913-1927 (2010)

11. R Szewczyk, A Mainwaring, J Polastre, J Anderson, D Culler, An analysis of a large scale habitat monitoring application, in Proceedings of the 2nd international conference on embedded networked sensor systems (SenSys04) (ACM Press, New York, 2004), pp. 214-226

12. S Lin, D Costello, M Miller, Automatic-repeat request error-control schemes. IEEE Commun. Mag. 22(12), 5-17 (1984)

13. T Le, W Hu, P Corke, S Jha, ERTP: energy-efficient and reliable transport protocol for data streaming in wireless sensor networks. Comput. Commun. 32(7), 1154-1171 (2009)

14. Z Rosberg, M Side, Selective-repeat ARQ: the joint distribution of the transmitter and the receiver resequencing buffer occupancies. IEEE Trans. Commun. 38(9), 1430-1438 (1990)

15. C Joo, NB Shroff, On the delay performance of in-network aggregation in lossy wireless sensor networks[J]. IEEE/ACM Trans. Netw. 22(2), 662-673 (2014)

16. L Keller, E Atsan, K Argyraki, C Fragouli, SenseCode: network coding for reliable sensor networks. ACM Trans. Sensor Networks. 9(2), 25 (2013)
17. Y Gao, J Bu, W Dong, C Chen, L Rao, X Liu, Exploiting concurrency for efficient dissemination in wireless sensor networks. IEEE Trans. Parallel Distrib. Syst. 24(4), 691-700 (2013)

18. K Pang, Z Lin, BF Uchoa-Filho, B Vucetic, Distributed network coding for wireless sensor networks based on rateless LT codes. IEEE Wireless Commun. Lett. 1(6), 561-564 (2012)

19. M Xu, W Song, Y Zhao, Collaborative data collection with opportunistic network erasure coding. IEEE Trans. Parallel Distrib. Syst. 24(10), 1941-1950 (2013)

20. P. Xie, J.H. Cui, On packet size and error correction optimisations in low-power wireless networks, in ICCCN (Proceedings IEEE, Piscataway, 2012), pp. 747-753

21. C. Noda, S. Prabh, M. Alves, T. Voigt, On packet size and error correction optimisations in low-power wireless networks, in SECON (Proceedings IEEE SECON, Piscataway, 2013), pp. 212-220

22. Y Liu, A Liu, Y Hu et al., FFSC: an energy efficiency communications approach for delay minimizing in internet of things. IEEE Access 4, 3775-3793 (2016)

23. YF Cai, YJ Mo, K Ota, CQ Luo, MX Dong et al., Optimal data fusion of collaborative spectrum sensing under attack in cognitive radio networks. IEEE Network 28(1), 17-23 (2014)

24. S Hussain, JH Park, AK Dey, LT Yang et al., Information fusion in future generation communication environments. Information Fusion 12(3), 148-149 (2012)

25. The OMNET++ simulator. [Online] Available: http://www.omnetpp.org/. Accessed 1 Feb 2015

26. H Wang, X Zhang, F Nait-Abdesselam, A Khokhar, Cross-layer optimized MAC to support multihop QoS routing for wireless sensor networks. IEEE Trans. Veh. Technol. 59(5), 2556-2563 (2010)

27. C Shanti, A Sahoo, DGRAM: a delay guaranteed routing and MAC protocol for wireless sensor networks. IEEE Trans. Mob. Comput. 9(10), 1407-1423 (2010)

28. A Liu, Y Hu, Z Chen, An energy-efficient mobile target detection scheme with adjustable duty cycles in wireless sensor networks. Int. J. Ad Hoc Ubiquitous Comput. 22(4), 203-225 (2016)

29 M Dong, K Ota, A Liu, RMER: reliable and energy efficient data collection for large-scale wireless sensor networks. IEEE Internet Things J. 3(4), 511-519 (2016)

30 H Li, D Liu, Y Dai, T Luan, Engineering searchable encryption of mobile cloud networks: when QoE meets QoP. IEEE Wirel. Commun. 22(4), 74-80 (2015)

31 H Li, Y Yang, T Luan, X Liang, L Zhou, X Shen, Enabling fine-grained multikeyword search supporting classified sub-dictionaries over encrypted cloud data. IEEE Trans. Dependable Secure Comput. 13(3), 312-325 (2016)

$32 \mathrm{H}$ Li, X Lin, H Yang, X Liang, R Lu, X Shen, EPPDR: an efficient privacypreserving demand response scheme with adaptive key evolution in smart grid. IEEE Trans. Parallel Distrib. Syst. 25(8), 2053-2064 (2014)

33 K Wei, S Guo, X Li, D Zeng, K Xu, Congestion control in social-based sensor networks: a social network perspective. Peer-to-Peer Netw. Appl. 9(4), 681-691 (2016)

$34 \mathrm{~L} \mathrm{Gu}$, D Zeng, S Guo, Y Xiang, J Hu, A general communication cost optimization framework for big data stream processing in geo-distributed data centers. IEEE Trans. Comput. 65(1), 19-29 (2016)

35 A Liu, Q Zhang, Z Li, Y Choi, J Li, N Komuro, A green and reliable communication modeling for industrial internet of things. Comput. Electr. Eng. (2016). doi:10.1016/j.compeleceng.2016.09.005

\section{Submit your manuscript to a SpringerOpen ${ }^{\circ}$ journal and benefit from:}

- Convenient online submission

- Rigorous peer review

- Immediate publication on acceptance

Open access: articles freely available online

- High visibility within the field

- Retaining the copyright to your article

Submit your next manuscript at $>$ springeropen.com 